\title{
Septal membrane localization by C-terminal amphipathic $\alpha$-helices of MinD in Bacillus subtilis mutant cells lacking MinJ or DivIVA
}

\author{
Kazuki Ishikawa ${ }^{\dagger}$ Satoshi Matsuoka, Hiroshi Hara and Kouji Matsumoto* \\ Department of Biochemistry and Molecular Biology, Graduate School of Science and Engineering, \\ Saitama University, 255 Shimo-ohkubo, Sakura-ku, Saitama-shi, Saitama 338-8570, Japan
}

(Received 6 October 2016, accepted 15 March 2017; J-STAGE Advance published date: 30 June 2017)

The Min system, which inhibits assembly of the cytokinetic protein FtsZ, is largely responsible for positioning the division site in rod-shaped bacteria. It has been reported that MinJ, which bridges DivIVA and MinD, is targeted to the cell poles by an interaction with DivIVA, and that MinJ in turn recruits MinCD to the cell poles. MinC, however, is located primarily at active division sites at mid-cell when expressed from its native promoter. Surprisingly, we found that Bacillus subtilis MinD is located at nascent septal membranes and at an asymmetric site on lateral membranes between nascent septal membranes in filamentous cells lacking MinJ or DivIVA. Bacillus subtilis MinD has two amphipathic $\alpha$-helices rich in basic amino acid residues at its C-terminus; one of these, named MTS1 here, is the counterpart of the membrane targeting sequence (MTS) in Escherichia coli MinD while the other, named MTS-like sequence (MTSL), is the nearest helix to MTS1. These amphipathic helices were located independently at nascent septal membranes in cells lacking MinJ or DivIVA, whereas elimination of the helices from the wild type protein reduced its localization considerably. MinD variants with altered MTS1 and MTSL, in which basic amino acid residues were replaced with proline or acidic residues, were not located at nascent septal membranes, indicating that the binding to the nascent septal membranes requires basic residues and a helical structure. The septal localization of MTSL, but not of MTS1, was dependent on host cell MinD. These results suggest that MinD is targeted to nascent septal membranes via its C-terminal amphipathic $\alpha$-helices in B. subtilis cells lacking MinJ or DivIVA. Moreover, the diffuse distribution of MinD lacking both MTSs suggests that only a small fraction of MinD depends on MinJ for its localization to nascent septal membranes.

Key words: amphipathic $\alpha$-helix, Bacillus subtilis, membrane targeting sequence, MinD, MinJ

\section{INTRODUCTION}

Cell division of rod-shaped bacteria is initiated by the assembly of the cytokinetic protein FtsZ, a homolog of eukaryotic tubulin, which polymerizes into a ring-like structure, the FtsZ-ring, at the division site and becomes the scaffold for assembly of the other division proteins (Bi and Lutkenhaus, 1991; Aarsman et al., 2005; Gamba et al., 2009). This division should take place at mid-cell

\footnotetext{
Edited by Kei Asai

* Corresponding author. E-mail: koumatsu@mail.saitama-u.ac.jp

$\dagger$ Present Address: Kyoto laboratory, arkray inc., Yousuien-nari, 59 Gansuin-cho, Kamigyo-ku, Kyoto 602-0008, Japan DOI: http://doi.org/10.1266/ggs.16-00054
}

between the future daughter chromosomes, and the selection of the correct site for division is controlled by two negative regulatory systems: nucleoid occlusion and the Min system. Nucleoid occlusion blocks Z-ring assembly over chromosomes and the Min system blocks such assembly at cell poles (Errington et al., 2003; Wu and Errington, 2004; Bernhardt and de Boer, 2005; Lutkenhaus, 2007; Rowlett and Margolin, 2015). The selection of the correct cell division site thus results from the inhibition of Z-ring assembly at all locations other than the appropriate site.

MinC and MinD form the complex MinCD that prevents the assembly of the Z-ring (de Boer et al., 1989, 1990). MinC is the inhibitor of FtsZ assembly (Dajkovic et al., 2008; Scheffers, 2008), whereas MinD has the role 
of linking MinC to membranes to which it binds itself via a C-terminal membrane targeting sequence (MTS) (Szeto et al., 2002; Hu and Lutkenhaus, 2003; Mileykovskaya et al., 2003). An intriguing aspect of the system in Escherichia coli is the oscillation of MinCD from pole to pole in the cells, which is controlled by a topological regulator, MinE, that also oscillates. Through this oscillation, the concentration of the inhibitor MinC is highest at the poles and lowest at mid-cell, which permits the assembly of the Z-ring at mid-cell (Raskin and de Boer, 1999; Fu et al., 2001; Hu and Lutkenhaus, 2001; Meinhardt and de Boer, 2001). In Bacillus subtilis, MinCD was thought to be located stably at the poles to form a bipolar gradient of MinC (Marston et al., 1998; Marston and Errington, 1999). However, a recent study reveals that high concentrations of MinC are found primarily at the septum and, more transiently, at the new poles and that MinC moves to mid-cell (the active division site) from new poles (recently completed septa) to prevent the formation of another Z-ring adjacent to the new poles (Gregory et al., 2008). Although the Min system was thought to act specifically on FtsZ assembly, it also blocks division downstream of FtsZ assembly, thereby acting at multiple levels to prevent inappropriate division (Bramkamp et al., 2008; van Baarle and Bramkamp, 2010). The correct localization of MinCD has been shown to be regulated by MinJ and DivIVA; DivIVA is first located at cell division sites and poles by being targeted to negatively curved membranes (Lenarcic et al., 2009; Ramamurthi and Losick, 2009) and then, by protein-protein interaction, MinJ also becomes located there, followed by MinD (Bramkamp et al., 2008; Patrick and Kearns, 2008).

In this study, we focus on MinD binding to the septal membranes in $B$. subtilis cells. It has been reported that B. subtilis MinD has an MTS with an amphipathic $\alpha$-helix at the C-terminus (Szeto et al., 2002, 2003; Hu and Lutkenhaus, 2003; Zhou and Lutkenhaus, 2003). One face of the $\alpha$-helix is highly hydrophobic and interacts with lipid acyl chains in the membrane, and the opposite face is polar and rich in basic amino acid residues apt to interact with the polar head group of acidic phospholipids on the membrane (Zhou and Lutkenhaus, 2003). A synthetic MTS of B. subtilis MinD has been shown to interact to form a stable $\alpha$-helix with acidic phospholipids in vitro (Szeto et al., 2003), suggesting that the acidic phospholipids, cardiolipin (CL) and phosphatidylglycerol (PG), on the membrane are involved in the action of MinD. The apparent contradiction between the interaction of the MTS of MinD with acidic phospholipids on the membrane and the recruitment by MinJ of MinD through proteinprotein interaction prompted us to reexamine the localization of MinD in B. subtilis cells. Here we report that B. subtilis MinD is targeted to the mid-cell site or septal membrane in the absence of MinJ and DivIVA. We also report that MTSs are responsible for this targeting, and that these MTSs comprise the known MTS (MTS1) and a newly identified, second amphipathic $\alpha$-helix (MTSL) located at the C-terminal region near to MTS1.

\section{MATERIALS AND METHODS}

Media and bacterial growth LB broth contained $1 \%$ tryptone (Difco), $0.5 \%$ yeast extract (Difco) and $1 \%$ $\mathrm{NaCl}$. The synthetic media CI and CII were used for development of transformation competence (Anagnostopoulos and Crawford, 1961). MacConkey agar medium (Nissui) was used for the $E$. coli two-hybrid experiment (Karimova et al., 2005). When required, the following supplements were added to the media (per liter): 50 or $100 \mathrm{mg}$ of spectinomycin (Sigma), $0.5 \mathrm{mg}$ of erythromycin (Sigma), $15 \mathrm{mg}$ of neomycin (Wako Pure Chemicals) and $10 \mathrm{mg}$ of tetracycline (Sigma) for B. subtilis, and $100 \mathrm{mg}$ of ampicillin (Sigma) and $50 \mathrm{mg}$ of kanamycin (Sigma) for $E$. coli. Xylose $(0.05 \%)$ was added at the time of inoculation (1/2,000-1/5,000 volume, to fresh LB medium) of an overnight culture, which was several hours before microscopic observation. Growth of bacteria was monitored by measuring turbidity with a Mini Photo 518R photometer (Taitec) equipped with a 530-nm interference filter and a 14-mm light path adaptor. For solid media, 1.5\% agar (Difco) was included.

Bacterial strains and plasmids Bacillus subtilis Marburg strains and plasmids used in this study are listed in Table 1 and Supplementary Table S1, respectively. For construction of gfp fusion strains and Min system-disrupted mutant strains, the following plasmid vectors were used. Vector pSG1729 (Lewis and Marston, 1999) allows fusions of gfp to the 5' end of a gene of interest, under the control of $\mathrm{P}_{x y l}$. Various mutant derivatives of minD genes were PCR-amplified using primer pairs designed as follows (cf. Supplementary Table S2). For construction of a GFP fusion to the N-terminus of a gene product, a sense primer that creates an in-frame fusion to the 3' end multicloning site of gfp on pSG1729 at the 5 ' end of the derivatives and an antisense primer at a position downstream of the termination codon or an inframe fusion to use the termination codon of the multicloning site, both with unique restriction endonuclease recognition sequences, were designed. PCR amplification was conducted using the Phusion High-Fidelity DNA polymerase PCR system (New England BioLabs), and gfp fusion plasmids were constructed by inserting each of the amplified products into the vectors. The gfp fusion allele on the plasmids was introduced into the chromosomal amyE locus of host strains, and its introduction was confirmed by disruption of $a m y E$ on LB plates containing $1.5 \%$ starch. The GFP-MinD we constructed was functional. Cells of strain KB221 (Pxyl-gfp-minD minD::pMUTINT3) produced minicells (5.8\%) in the 
Table 1. Bacterial strains used in this study

\begin{tabular}{|c|c|c|}
\hline Strain & Relevant characteristics & Source/Construct $^{*}$ \\
\hline \multicolumn{3}{|c|}{ B. subtilis } \\
\hline 168 & $\operatorname{trp} C 2$ & Laboratory stock \\
\hline YVJDd & $\operatorname{trp} C 2$ minJ::pMUTIN T3 erm & Kobayashi et al., 2003 \\
\hline KB021 & $\operatorname{trp} C 2 \min D:: \mathrm{pMUTIN}$ T3 erm & $168 \leftarrow$ pM-MinD \\
\hline KB026 & trpC2 divIVA::pMUTIN T3 erm & $168 \leftarrow$ pM-divIVA \\
\hline KB027 & $\operatorname{trp} C 2 \min C:$ :pMUTIN T3 erm Pspac-minD & $168 \leftarrow$ pM-MinC \\
\hline KB028 & $\operatorname{trp} C 2 \min C:: \mathrm{pMUTIN}$ T3 tet $\mathrm{P} s p a c-\min D$ & $\mathrm{~KB} 027 \leftarrow \mathrm{pEm}:: \mathrm{Tc}$ \\
\hline KB029 & KB028 minJ::pMUTIN T3 erm & KB028 $\leftarrow$ YVJDd DNA \\
\hline KB030 & KB028 divIVA::pMUTIN T3 erm & $\mathrm{KB} 028 \leftarrow \mathrm{KB} 026 \mathrm{DNA}$ \\
\hline SU561 & $\operatorname{trp} C 2 \min C D:: c a t$ & Rodrigues \& Harry, 2012 \\
\hline MB001 & $\operatorname{trp} C 2 \min C D::$ cat minJ::pMUTINT3 erm & SU561 $\leftarrow$ YVJDd DNA \\
\hline MB002 & $\operatorname{trp} C 2 \min C D:: c a t$ divIVA::pMUTINT3 erm & SU561 $\leftarrow$ KB026 DNA \\
\hline JN19 & $\operatorname{trpC2}$ amyE::Pxyl-gfp & Kusaka et al., 2016 \\
\hline KB200 & $\operatorname{trpC} 2$ amyE::Pxyl-gfp-minD & $168 \leftarrow$ pGFP-MinD \\
\hline KB211 & trpC2 divIVA::pMUTINT3 erm amyE::Pxyl-gfp-minD & $\mathrm{KB} 026 \leftarrow \mathrm{KB} 200 \mathrm{DNA}$ \\
\hline KB221 & $\operatorname{trp} C 2$ minD::pMUTINT3 erm amyE::Pxyl-gfp-minD & $\mathrm{KB} 021 \leftarrow \mathrm{KB} 200 \mathrm{DNA}$ \\
\hline KB224 & trpC2 minD-gfp erm & $168 \leftarrow \mathrm{pMm} 2-\mathrm{MinD}$ \\
\hline KB225 & $\operatorname{trpC2}$ amyE::Pxyl-gfp-MTSL & $168 \leftarrow$ pGFP-MTSL \\
\hline KB226 & trpC2 amyE::Pxyl-gfp-MTS1 & $168 \leftarrow$ pGFP-MTS1 \\
\hline KB227 & $\operatorname{trpC2}$ amyE::Pxyl-gfp-MTSL-MTS1 & $168 \leftarrow$ pGFP-MTSL-MTS1 \\
\hline KB229 & $\operatorname{trpC2}$ amyE::Pxyl-gfp-minD${ }^{\Delta \mathrm{MTS}}$ & $168 \leftarrow$ pGFP-MinD ${ }^{\Delta \mathrm{MTS}}$ \\
\hline KB236 & $\operatorname{trpC2}$ amyE::Pxyl-gfp-minD ${ }^{\mathrm{M} 1-\mathrm{G} 241}$ & $168 \leftarrow$ pGFP-MinD ${ }^{\Delta \mathrm{MTS} 1}$ \\
\hline KB237 & KB021 amyE::Pxyl-gfp-minD ${ }^{\Delta \mathrm{MTS}}$ & $\mathrm{KB} 021 \leftarrow$ pGFP-MinD ${ }^{\Delta \mathrm{MTS}}$ \\
\hline KB238 & KB021 amyE::Pxyl-gfp-minD ${ }^{\mathrm{M} 1-\mathrm{G} 241}$ & $\mathrm{~KB} 021 \leftarrow$ pGFP-MinD ${ }^{\Delta \mathrm{MTS} 1}$ \\
\hline KB240 & $\operatorname{trpC2}$ amyE::Pxyl-gfp-minD ${ }^{\Delta(\mathrm{P} 224-\mathrm{G} 241)}$ & $168 \leftarrow$ pGFP-MinD ${ }^{\Delta M T S L}$ \\
\hline KB244 & $\operatorname{trpC2}$ amyE::Pxyl-gfp-minD${ }^{\mathrm{M1} 1 \mathrm{G} 241}$-MTSL & $168 \leftarrow$ pGFP-MinD ${ }^{\Delta \mathrm{MTS} 1}$-MTSL \\
\hline KB245 & $\operatorname{trpC} 2$ amyE::Pxyl-gfp-minD ${ }^{\Delta(\mathrm{P} 224-\mathrm{G} 241)}$-MTSL & $168 \leftarrow$ pGFP-MinD ${ }^{\Delta \mathrm{MTSL}}$ \\
\hline KB246 & $\operatorname{trpC2}$ amyE::Pxyl-gfp-minD ${ }^{\Delta(\mathrm{P} 224-\mathrm{G} 241)}$-MTS1 & $168 \leftarrow$ pGFP-MinD ${ }^{\triangle M T S L}$-MTS1 \\
\hline KB249 & KB021 amyE::Pxyl-gfp-minD ${ }^{\Delta(\mathrm{P} 224-\mathrm{G} 241)}$ & 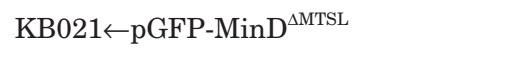 \\
\hline KB253 & KB021 amyE::Pxyl-gfp-min ${ }^{\text {M1-G241-MTSL }}$ & KB021 $\leftarrow$ pGFP-MinD ${ }^{\Delta \mathrm{MTS} 1}$-MTSL \\
\hline KB254 & KB021 amyE::Pxyl-gfp-minD ${ }^{\Delta(\mathrm{P} 224-\mathrm{G} 241)}$-MTSL & KB021 $\leftarrow$ pGFP-MinD ${ }^{\Delta M T S L}$-MTSL \\
\hline KB255 & KB021 amyE::Pxyl-gfp-minD ${ }^{\Delta(\mathrm{P} 224-\mathrm{G} 241)}$-MTS1 & KB021 $\leftarrow$ pGFP-MinD ${ }^{\Delta \mathrm{MTSL}(224-241)}$-MTS1 \\
\hline KB257 & KB021 amyE::Pxyl-gfp-minD ${ }^{\Delta(\mathrm{P} 224-\mathrm{L} 246)}$-MTS1 & KB021 $\leftarrow$ pGFP-MinD ${ }^{\Delta \mathrm{MTSL}(224-246)}$-MTS1 \\
\hline KB258 & $\operatorname{trpC2}$ amyE::Pxyl-gfp-minD${ }^{\mathrm{R} 237 \mathrm{P}}$ & $168 \leftarrow$ pGFP-MinD ${ }^{\mathrm{R} 237 \mathrm{P}}$ \\
\hline KB259 & $\operatorname{trpC2}$ amyE::Pxyl-gfp-minD ${ }^{\mathrm{K} 259 \mathrm{P}}$ & $168 \leftarrow$ pGFP-MinD ${ }^{\mathrm{K} 259 \mathrm{P}}$ \\
\hline KB260 & $\operatorname{trpC2} a m y E:: \mathrm{P} x y l-g f p-m i n D^{\mathrm{R} 37 \mathrm{P} / \mathrm{K} 259 \mathrm{P}}$ & $168 \leftarrow$ pGFP-MinD ${ }^{\text {R237P/K259P }}$ \\
\hline KB261 & $\operatorname{trp} C 2$ amyE::Pxyl-gfp-minD MTSL ${ }^{(\mathrm{R} 237 \mathrm{P})}$ & $168 \leftarrow$ pGFP-MTSL $^{\text {R237P }}$ \\
\hline KB262 & $\operatorname{trp} C 2$ amyE::Pxyl-gfp-minD $\mathrm{MTS}^{(\mathrm{K} 259 \mathrm{P})}$ & $168 \leftarrow$ pGFP-MTS $1^{\text {K259P }}$ \\
\hline KB263 & KB021 amyE::Pxyl-gfp-minD ${ }^{\mathrm{R} 237 \mathrm{P}}$ & $\mathrm{KB} 021 \leftarrow$ pGFP-MinD ${ }^{\mathrm{R} 237 \mathrm{P}}$ \\
\hline KB264 & KB021 amyE::Pxyl-gfp-minD ${ }^{\mathrm{K} 259 \mathrm{P}}$ & $\mathrm{KB} 021 \leftarrow \mathrm{pGFP}-\mathrm{MinD}^{\mathrm{K} 259 \mathrm{P}}$ \\
\hline KB265 & KB021 amyE::Pxyl-gfp-minD ${ }^{\mathrm{R} 237 \mathrm{P} / \mathrm{K} 259 \mathrm{P}}$ & KB021 $\leftarrow$ pGFP-MinD ${ }^{\text {R237P/K259P }}$ \\
\hline KB279 & $\operatorname{trpC2}$ amyE::Pxyl-gfp-minD MTSL ${ }^{\text {acidic }}$ & $168 \leftarrow$ pGFP-MTSL ${ }^{\text {acidic }}$ \\
\hline KB283 & $\operatorname{trp} C 2$ amyE ::Pxyl-gfp-minD MTS1 $1^{\text {acidic }}$ & $168 \leftarrow$ pGFP-MTS $1^{\text {acidic }}$ \\
\hline KB267 & KB029 amyE::Pxyl-gfp-minD & KB029 $\leftarrow$ pGFP-MinD \\
\hline KB268 & KB029 amyE::Pxyl-gfp-MTSL & KB029 $\leftarrow$ pGFP-MTSL \\
\hline
\end{tabular}




\begin{tabular}{|c|c|c|}
\hline KB269 & KB029 amyE::Pxyl-gfp-MTS1 & KB029 $\leftarrow$ pGFP-MTS1 \\
\hline KB270 & KB029 amyE::Pxyl-gfp-minD ${ }^{\mathrm{M} 1-\mathrm{D} 223}$ & KB029 $\leftarrow$ pGFP-MinD ${ }^{\triangle M T S}$ \\
\hline KB273 & KB030 amyE::Pxyl-gfp-minD & $\mathrm{KB} 030 \leftarrow$ pGFP-MinD \\
\hline KB274 & KB030 amyE::Pxyl-gfp-minD ${ }^{\text {M1-D223 }}$ & $\mathrm{KB} 030 \leftarrow$ pGFP-MinD ${ }^{\Delta \mathrm{MTS}}$ \\
\hline KB275 & KB030 amyE::Pxyl-gfp-minD MTSL & KB030 $\leftarrow$ pGFP-MTSL \\
\hline KB276 & KB030 amyE::Pxyl-gfp-minD MTS1 & KB030 $\leftarrow$ pGFP-MTS1 \\
\hline KB281 & KB021 amyE::Pxyl-gfp-MTSL-MTS1 & KB021 $\leftarrow$ pGFP-MTSL-MTS1 \\
\hline KB313 & trpC2 divIVA-gfp Pspac-divIVA erm & $168 \leftarrow$ pMm2-DivIVA' \\
\hline KB400 & $\operatorname{trpC2}$ minJ-gfp erm & $168 \leftarrow \mathrm{pMm} 2-\mathrm{MinJ}$ \\
\hline KB508 & $\operatorname{trp} C 2$ minC-gfp erm Pspac-minD & $168 \leftarrow \mathrm{pMm} 2-\mathrm{MinC}$ \\
\hline MB500 & trpC2 minJ::pMUTINT3 erm amyE::Pxyl-gfp-minD & YVJDd $\leftarrow$ KB200 DNA \\
\hline DS2945 & $3610 \Delta$ minJ amyE:: $\mathrm{P}_{\text {minJ }}-$ minJ cat & Patrick \& Kearns, 2008 \\
\hline MB531 & $3610 \Delta$ minJ amyE::Pxyl-gfp-minD & $\mathrm{DS} 2945 \leftarrow \mathrm{KB} 200$ \\
\hline MB511 & MB001 amyE::Pxyl-gfp-minD & $\mathrm{MB} 001 \leftarrow \mathrm{KB} 200 \mathrm{DNA}$ \\
\hline MB512 & MB001 amyE::Pxyl-gfp-MTSL & $\mathrm{MB} 001 \leftarrow \mathrm{KB} 225 \mathrm{DNA}$ \\
\hline MB513 & MB001 amyE::Pxyl-gfp-MTS1 & $\mathrm{MB} 001 \leftarrow \mathrm{KB} 226 \mathrm{DNA}$ \\
\hline MB514 & MB001 amyE::Pxyl-gfp-minD ${ }^{\text {M1-D223 }}$ & 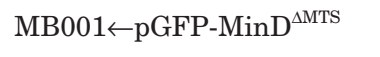 \\
\hline MB541 & MB002 amyE::Pxyl-gfp-minD & $\mathrm{MB} 002 \leftarrow \mathrm{KB} 200 \mathrm{DNA}$ \\
\hline MB542 & MB002 amyE::Pxyl-gfp-MTSL & $\mathrm{MB} 002 \leftarrow \mathrm{KB} 225 \mathrm{DNA}$ \\
\hline MB543 & MB002 amyE::Pxyl-gfp-MTS1 & $\mathrm{MB} 002 \leftarrow \mathrm{KB} 226 \mathrm{DNA}$ \\
\hline MB544 & MB002 amyE::Pxyl-gfp-minD ${ }^{\text {M1-D223 }}$ & MB002 $\leftarrow$ pGFP-MinD ${ }^{\Delta M T S}$ \\
\hline \multicolumn{3}{|l|}{ E. coli } \\
\hline DH5 & recA1 gyrA96 supE44 hsdR17 relA1 & Laboratory stock \\
\hline $\mathrm{C} 600$ & supE44 hsdR thi-1 thr-1 leuB6 lacY tonA21 & Sadaie, Y. \\
\hline CH101 & $\mathrm{F}^{-}$cya-99 araD139 galE15 galK16 rpsL1 $\left(\mathrm{Str}^{\mathrm{r}}\right)$ hsdR2 mcrA1 mcrB1 & Euromedex \\
\hline
\end{tabular}

* Transformation with chromosomal or plasmid DNA.

absence of xylose, but supplementation with the inducer ( $0.05 \%$ xylose) reduced the level of minicell production to $0.13 \%$, which corresponded to that of wild type 168 cells. This indicates that Pxyl-gfp-minD is fully functional and that $0.05 \%$ xylose is sufficient for suppression of the minicell production phenotype of the $\min D$ mutation. The products of these gfp fusion genes were examined by Western blotting with anti-GFP antibody, and it was confirmed that all the fusion constructs were stable and produced an amount of GFP fusion products almost equal to the amounts produced by GFP fusions to wild type MinD. Plasmid pMm2 (Takamatsu et al., 2000) was used to construct a strain expressing a $g f p$ fusion gene under its own promoter on the chromosome as previously described (Nishibori et al., 2005). The minJ-gfp construct (KB400) was fully functional as the strain produced only small numbers of minicells, comparable to the level of wild type 168, but the constructs with $\min C$-gfp, minD-gfp and divIVA-gfp (KB508, KB224 and KB313, respectively) were not functional, as they produced minicells. Test pairs of plasmids of adenylate cyclase domains, T18-MinC, MinC-T18, T18-MinD and T25-fused MinD possessing various mutant MTSs were constructed on the vectors pUT18, pUT18C and pKT25 (Karimova et al., 2005), respectively. Recombinant DNA procedures were based on standard methods (Sambrook et al., 1989).

Fluorescence microscopy For localization of the images of GFP fusions and membranes stained with FM 4-64, fluorescence microscopy was conducted as described previously (Nishibori et al., 2005). Bacillus subtilis cells harboring $g f p-\min D$ with or without C-terminal $\alpha$-helical domains, $g f p$-minD with altered MTS sequence, or $g f p$ fused with a sequence containing the MTS alone were cultivated in LB medium containing $0.05 \%$ xylose up to mid- and late-logarithmic growth phase. FM 4-64 (Invitrogen) and DAPI (4',6-diamidino-2-phenylindole) (Dojindo) were added, to a concentration of $1 \mu \mathrm{g} / \mathrm{ml}$, and incubated for $5 \mathrm{~min}$. Cells in culture were concentrated by a brief centrifugation and fixed on object slides coated with a layer of $1 \%(\mathrm{wt} / \mathrm{vol})$ agarose in water, and were then subjected to fluorescence microscopy. Fluorescence images were viewed with an ECLIPSE E600 fluorescence microscope (Nikon) and a cooled CCD camera (ORCA-ER; Hamamatsu Photonics). Red fluorescence from FM 4-64 was detected using a G-2A filter unit. Green fluorescence 
from GFP was detected with a standard GFP(R)-BP filter unit. To minimize the toxicity of high-energy excitation light, the focus was set under phase-contrast conditions and the fluorescence images were captured shortly after the shift to high-energy excitation light. The exposure time for FM 4-64 was 0.4-0.8 sec, that for green fluorescence of GFP was 1.7-4 sec, that for DAPI was $0.2-0.6$ sec, and that for phase contrast observation was 0.004 0.01 sec. Captured images were processed with Adobe Photoshop 6.0.

\section{RESULTS}

MinD is located at nascent septal membranes in minJ-disrupted and divIVA-disrupted cells Reports indicate that disruption of the new essential member MinJ in the Min system of B. subtilis results in GFPMinD disappearing from its normal polar and division site locations. It is, instead, dispersed throughout the cytoplasm or concentrated in foci that are randomly scattered throughout the filament of cells (Bramkamp et al., 2008), or forms randomly distributed, membrane- associated puncta (Patrick and Kearns, 2008). Thus MinJ is required for the polar localization of MinD in B. subtilis cells. In an $E$. coli two-hybrid analysis, MinJ has been found to interact directly with DivIVA and MinD (Bramkamp et al., 2008; Patrick and Kearns, 2008). Therefore, these data support the hypothesis that MinJ acts as an adaptor in delivering MinD to DivIVA at cell poles by protein-protein interaction. This hypothesis does not take into account the fact that MinD has an MTS at its C-terminus that directly interacts with acidic phospholipids in the membrane, as evidenced by in vitro studies of both $B$. subtilis and $E$. coli ( $\mathrm{Hu}$ and Lutkenhaus, 2003; Mileykovskaya et al., 2003; Szeto et al., 2003). This led us to reexamine the mode of action of $\mathrm{MinD}$ in the $B$. subtilis Min system.

First, to examine the location of MinD in cells lacking MinJ we introduced $\mathrm{P}_{x y l}$-gfp-minD, which was on the integration vector pSG1729 (Lewis and Marston, 1999), into the amyE locus of the mutant strain YVJDd, which contains pMUTIN3-disrupted minJ ( $y v j D$ ) (Kobayashi et al., 2003), and into the $a m y E$ locus of the wild type strain as a control. The minJ-disrupted cells (strain MB500) were
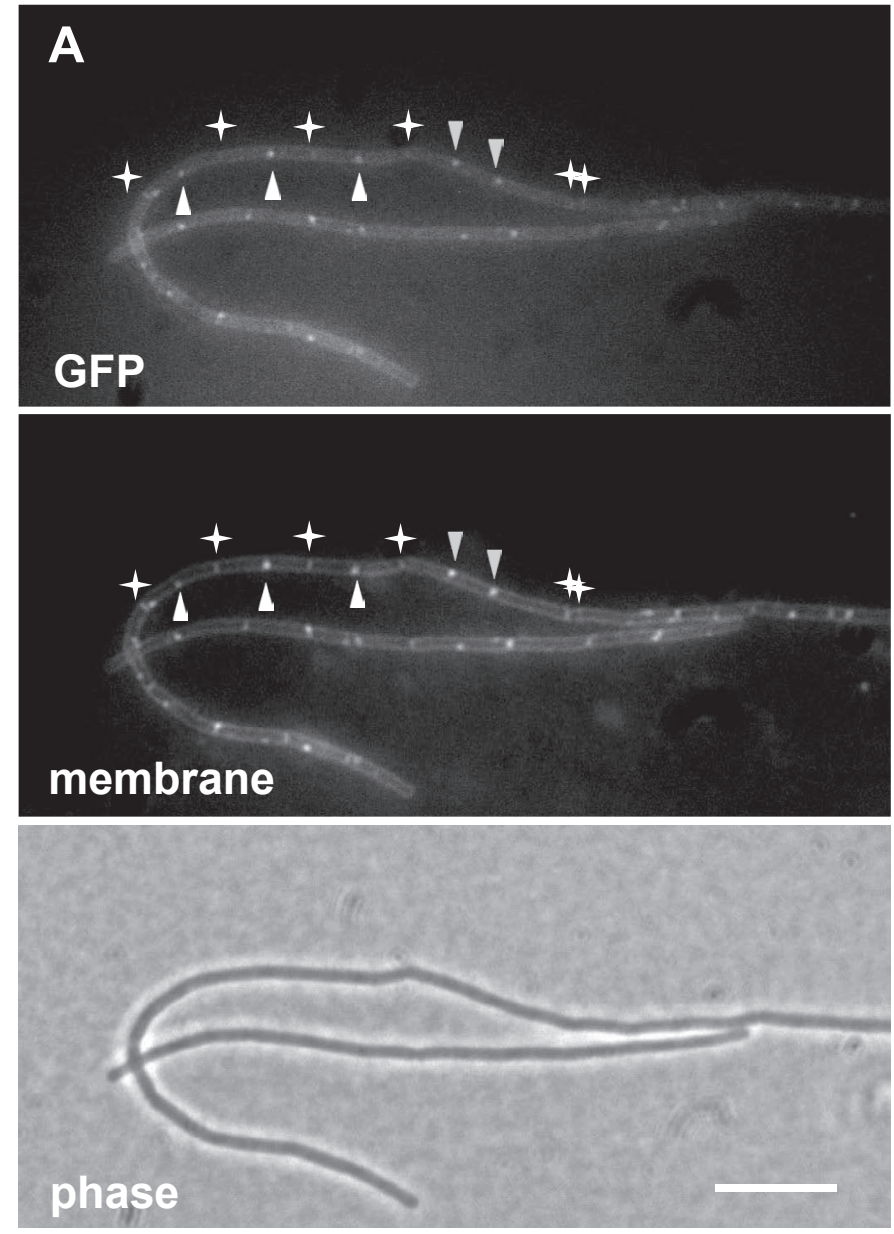
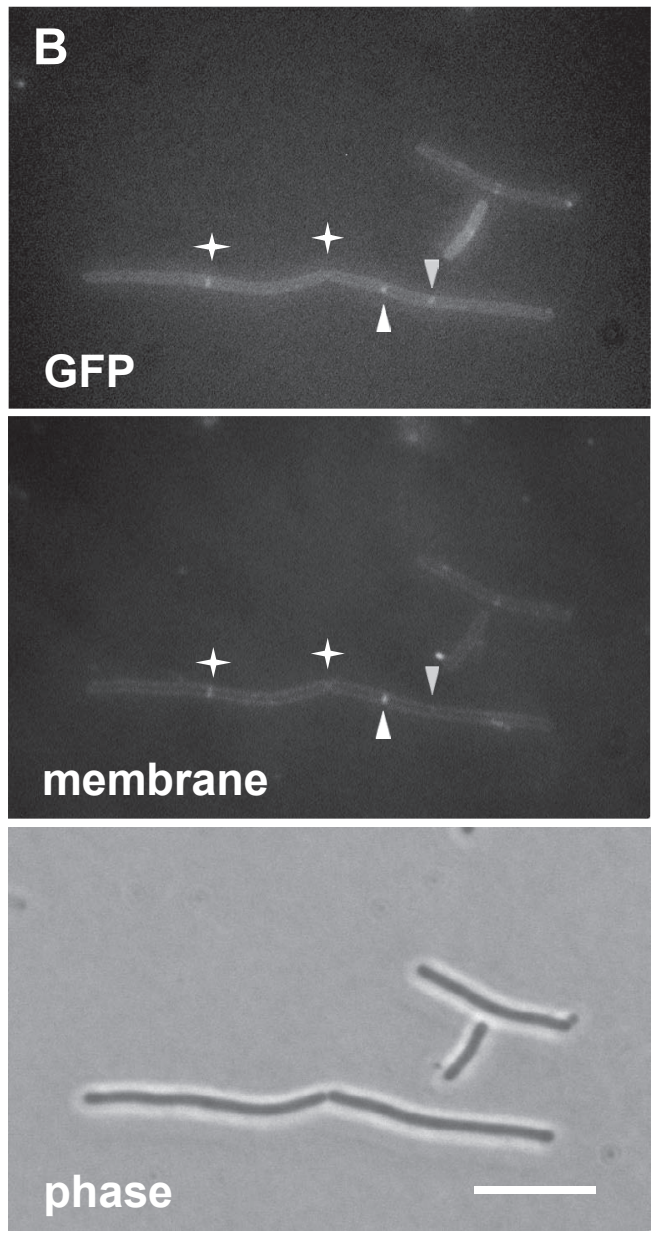

Fig. 1. Continued 

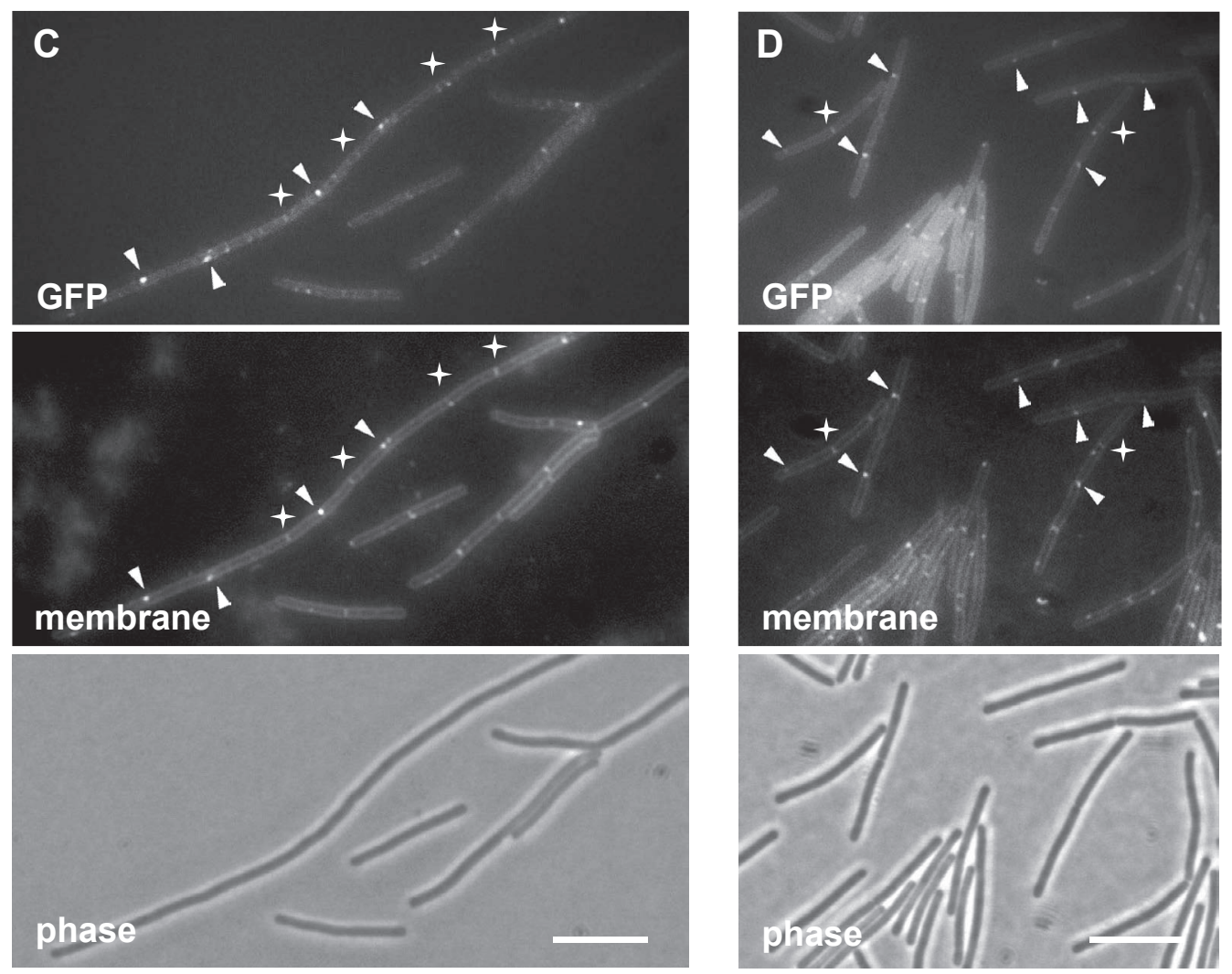
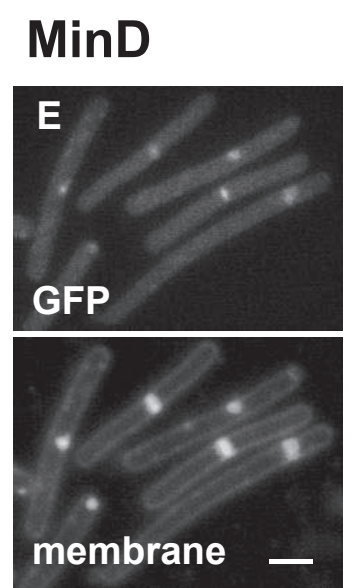

MinC

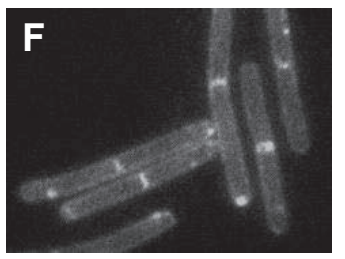

MinJ
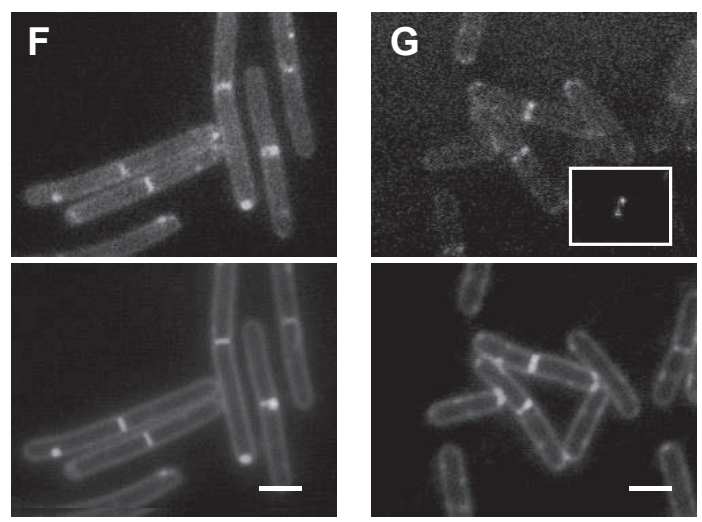

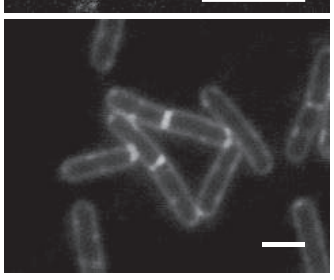

DivIVA

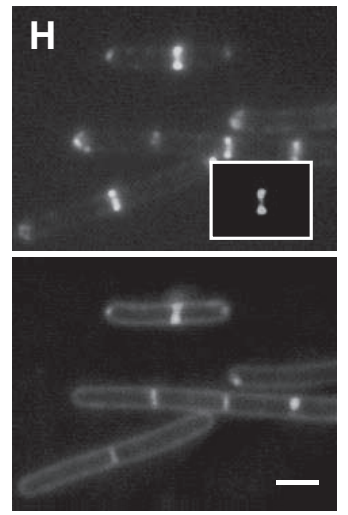

Fig. 1. Localization of MinD in minJ and divIVA mutant cells and of Min components in cells with wild type background. Cells of $B$. subtilis minJ and divIVA strains (A-D) harboring $\mathrm{P}_{x y l}$-gfp-minD fusions in the amyE locus were cultivated in LB medium containing $0.05 \%$ xylose to logarithmic growth phase, and Min system component-GFP strains harboring fusions under their own promoters in wild type background (E-H) were cultivated in LB medium. FM 4-64 was added to a final concentration of $1 \mu \mathrm{g} / \mathrm{ml}$ and the cells were incubated for $5 \mathrm{~min}$. The cells were harvested and subjected to fluorescence microscopy as described in Materials and Methods. The top panels in the three-panel groups (A-D) show GFP fluorescence, while the middle and bottom panels show membranes stained with FM 4-64 and phase contrast, respectively. A, minJ $\mathrm{P}_{x y l}$-gfp-minD (strain MB500); B, $\Delta$ minJ $\mathrm{P}_{x y l}$-gfp-minD (strain MB531); C, divIVA $\mathrm{P}_{x y l}$-gfp-minD (KB211); D, min $J^{+} \mathrm{P}_{x y l}$-gfp-minD (KB200). The gfp fusion allele of minD, Pxyl-gfp-minD, was functional: supplementation with $0.05 \%$ xylose fully suppressed the minicell production of $\min D$ mutants (see Materials and Methods). Arrowheads and stars indicate typical asymmetric foci and nascent septal membranes, respectively. Bars, $10 \mu \mathrm{m}$. The distances between nascent septal membranes where GFP-MinD localized were quantified; see Supplementary Fig. S1 for this information. The asymmetric foci are probably a consequence of the recycling of MinD from new polar membranes passing through lateral ones to assemble in nascent septal membranes, as shown for MinC (Gregory et al., 2008). The upper and lower panels in the paired images (E-H) show GFP fluorescence and membrane staining with FM 4-64, respectively. E, minD-gfp (KB224); F, minC-gfp (KB508); G, minJ-gfp (KB400); and $\mathrm{H}$, divIVA-gfp (KB313). IPTG $(1 \mathrm{mM})$ was supplemented for cultivation in LB medium of strains KB508 (F) and KB313 (H). The fractions of cells in which MinD-GFP, MinC-GFP, MinJ-GFP and DivIVA-GFP located at septal membranes were 33\%, 60\%, 72\% and $78 \%$, respectively, of the total cells counted (70-140). Bars, $2 \mu \mathrm{m}$. Insets in panels $\mathrm{G}$ and $\mathrm{H}$ show representative areas with reduced brightness to indicate a pair-of-dots signal facing the lateral membranes, which is interpretable as the two-dimensional projection of a three-dimensional open ring that is not filled with fluorescent materials. 
markedly impaired for division, forming long aseptate filaments with rare division sites, and produced minicells at the ends of the filaments, which was evident in midlogarithmic growth phase (Fig. 1A, compare with those of the wild type cells in Fig. 1D and Fig. 2B) as previously described for pMUTIN-disrupted and null minJ mutant cells by Bramkamp et al. (2008) and Patrick and Kearns (2008), respectively. GFP-MinD in the minJ-disrupted filamentous cells was mainly located at nascent septal membranes and as a focus at an asymmetric site on lateral membranes between the nascent septal membranes, forming discrete structures at fairly regular intervals along the cell filaments.

This pattern of MinD localization is consistent with the reported septal but not polar location of GFP-labeled MinC, as well as MinD, in cells where it is expressed from

A

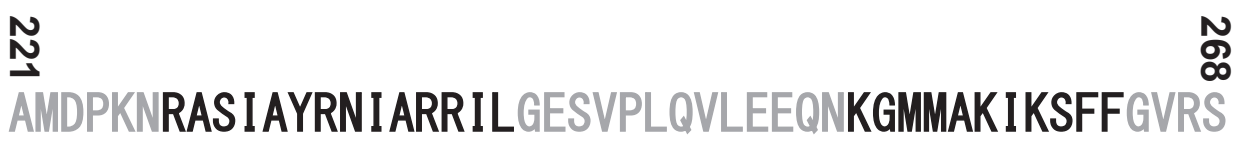

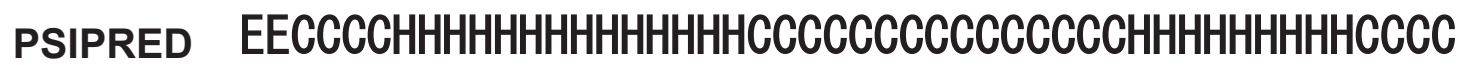

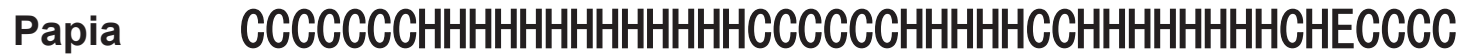
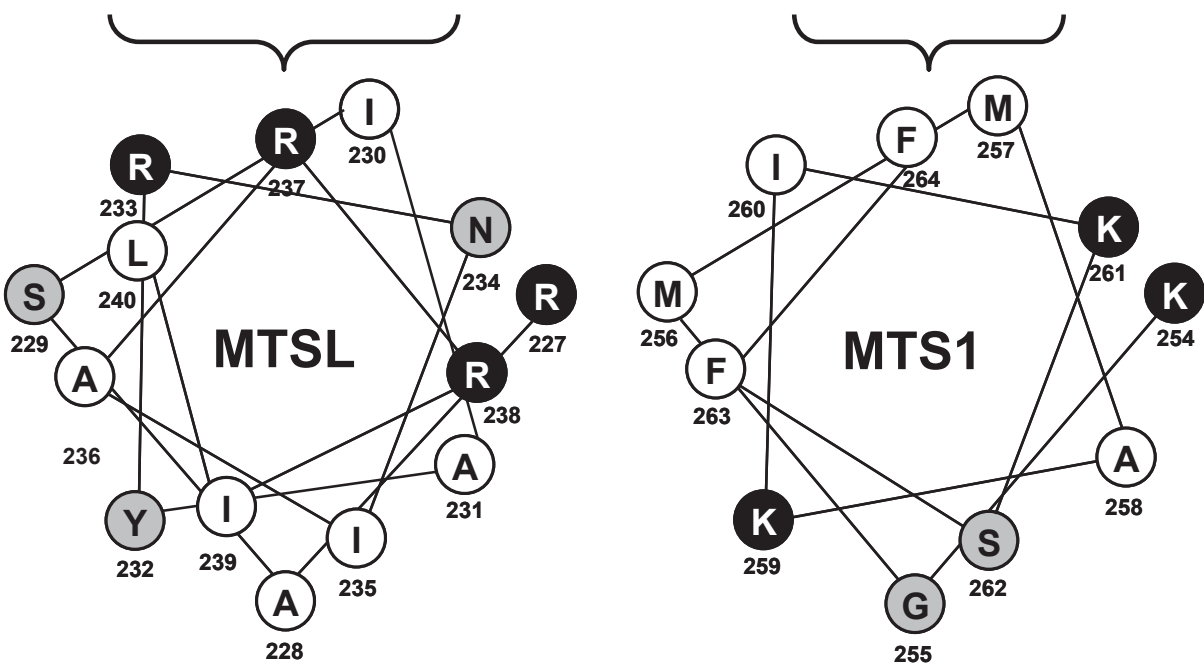

Full length M1-S268

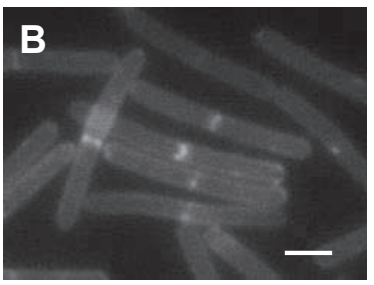

MTSL P224-G241

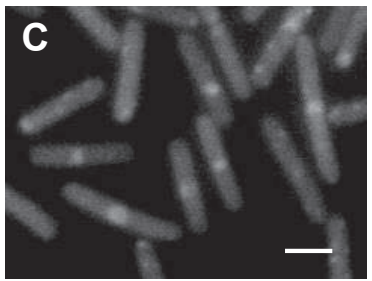

MTS1 Q252-S268

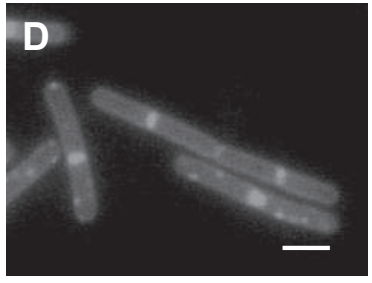

$\triangle \mathrm{MTS}$ M1-D223

Fig. 2. Predicted amphipathic $\alpha$-helix domains at the $\mathrm{C}$ terminus of B. subtilis $\mathrm{MinD}$ and their cellular localization. A, The predicted secondary structures (upper) of the C-terminal region (A221-S268) of MinD calculated by PSIPRED and Papia programs. H and C indicate predicted $\alpha$-helix and coil structure, respectively. Edmundson wheel analysis (lower) suggests a new C-terminal amphipathic $\alpha$-helix, MTSL (R227-L240), in addition to the known amphipathic $\alpha$-helix MTS1 (K254-F264), previously designated as the membrane targeting sequence (MTS) (Szeto et al., 2002). In the wheel analysis diagrams, the characteristics of the amino acid residues are indicated as follows: basic, polar and hydrophobic residues are named in black, gray and white circles, respectively. (B-E), localization of MinD (full length), the sequence P224-G241 containing MTSL and the sequence Q252-S268 containing MTS1 of MinD, and the variant ( $\triangle \mathrm{MTS}$ ) of MinD lacking both MTSs in wild type cells. Cells of B. subtilis 168 strains harboring gfp fusions in the amyE locus, listed below, were cultivated in LB media containing $0.05 \%$ xylose to late logarithmic growth phase. The cells were harvested and subjected to fluorescence microscopy as described in Materials and Methods. B, GFP-MinD (full length; strain KB200); C, GFP-(P224-G241) (MTSL; strain KB225); D, GFP-(Q252-S268) (MTS1; strain KB226); and E, GFP-MinD ${ }^{\triangle M T S}$ ( $\triangle$ MTS; strain KB229). Percentages of cells that showed septal membrane localization of GFP-labeled variants among the total cells counted (110-210): B, 40\%; C, 29\%; D, 34\%; E, weak septal, $35 \%$. Bars, $2 \mu \mathrm{m}$. 
the native promoter and in those where it is induced with $0.05 \%$ xylose or less (Gregory et al., 2008); this pattern therefore differs from the polar localization of MinD that had previously been reported with a much higher concentration $(0.5 \%)$ of the inducer (Marston et al., 1998; Marston and Errington, 1999). The GFP-labeled MinC expressed from the native promoter has been shown to be located at nascent septal membranes before visible constriction of the septal membrane, often forming a pair of foci at the future division site as evidenced by time-lapse microscopy (Gregory et al., 2008).

In the filaments of minJ-disrupted cells, GFP-MinD was mainly located at nascent septal membranes (Fig. 1A, indicated by stars; see the GFP panel and the middle one, which shows membranes stained with FM 4-64) and as asymmetric foci at a mid-cell site on lateral membranes between the two nascent septal membranes (indicated by arrowheads). The average interval between the nascent septal, including polar, membranes was $6.6 \pm 2.0 \mu \mathrm{m}$; that is, the deviation of the interval distances of nascent septal membranes was small (Supplementary Fig. S1A). This average distance was close to that observed for wild type cells (KB200): $7.0 \pm 1.8 \mu \mathrm{m}$ in mid-logarithmic growth phase. The formation of septal membranes is thus fairly regular, irrespective of the presence or absence of MinJ. The nascent septal membranes and the asymmetric foci of GFP-MinD in the minJ-disrupted cells were located in the inter-nucleoid space, as demonstrated by nucleoid staining with DAPI (Supplementary Fig. S2).

This localization of GFP-MinD at nascent septal membranes and as an asymmetric focus at a mid-cell site on lateral membranes was also observed in the MB531 strain harboring a $\Delta$ minJ mutation, which was constructed by introduction of the Pxyl-gfp-minD allele into a 3610-derived strain, DS2945, a gift of Dr. D. B. Kearns (Fig. 1B). These results are markedly different from the reported distribution of GFP-MinD fluorescence throughout the cytoplasm or in foci that are randomly scattered throughout the filament of minJ-disrupted cells or in random, membrane-associated puncta in null minJ mutant cells (Bramkamp et al., 2008; Patrick and Kearns, 2008). In those experiments, the $\mathrm{P}_{x y l}$ promoter was induced at a high level with $0.5 \%$ xylose. In our minJ-disrupted mutant cells, induction was usually performed with $0.05 \%$ xylose $(2-3 \mathrm{~h})$. When we tried highlevel expression with $0.5 \%$ xylose, this caused formation of many GFP-MinD foci along lateral membranes and a partly scattered distribution of foci throughout the cell filaments (Supplementary Fig. S3).

It is noteworthy that the principal location of the components of the Min system is on the nascent septal membranes at mid-cell. This is the case for MinC as well as for MinD when they are expressed from their native promoters (Gregory et al., 2008) and for MinJ expressed from its native promoter (van Baarle and Bramkamp,
2010). GFP-MinD in wild type cells (KB200) mainly localized at septal membranes (Fig. 1D). The fraction of septal localization was $36 \%$ of the total cells (55) in midlogarithmic growth induced for $2 \mathrm{~h}$ with $0.05 \%$ xylose, and the number of cells with polar localization was small $(3.6 \%)$. In the late logarithmic growth phase, induced for $4.5 \mathrm{~h}$, the cells with septal localization decreased to $15 \%$ and polar localization increased to $49 \%$ of the total cells (106) counted. At $0.5 \%$ xylose, induced for $4.5 \mathrm{~h}$ to midlogarithmic growth phase, the fraction of septal, polar and polar plus septal was $12 \%, 32 \%$ and $52 \%$, respectively of the cells (115) counted (Supplementary Fig. S3 B1), and after induction for $6 \mathrm{~h}$ to late logarithmic growth phase these fractions were $3 \%, 69 \%$ and $27 \%$, respectively, of the cells (135) counted (Supplementary Fig. S3 B2).

To re-confirm the natural locations of the components of the Min system, we constructed GFP fusion strains of the Min system components expressed from their own native promoters. MinD-GFP, MinC-GFP, MinJ-GFP and DivIVA-GFP were located mainly on the septal membranes (Fig. 1E-H), although MinD-GFP showed a diffused band. In addition, MinD-GFP formed many foci on lateral membranes (31\%). Its diffusion and the low level of septal membrane localization as well as the many foci on lateral membranes may be due to a functional defect caused by the fusion of GFP at the C-terminus, which has an essential role for its septal membrane localization (see below). The distribution of MinJ and DivIVA as a pair of dots facing lateral membranes is clear (Fig. $1 \mathrm{G}$ and $\mathrm{H}$, insets), and this is interpretable as the two-dimensional projection of a three-dimensional open ring that is not filled with fluorescent material.

Furthermore, in cells lacking DivIVA, GFP-MinD was also distributed on nascent septal membranes and formed asymmetric foci on lateral membranes (Fig. 1C, stars and arrowheads, respectively). The average distance between the nascent septal membranes in divIVA cells was $7.4 \pm$ $2.3 \mu \mathrm{m}$ (Supplementary Fig. S1B), close to that of wild type cells (KB200), which had an average distance of $7.0 \pm 1.8 \mu \mathrm{m}$ in mid-logarithmic growth phase. Neither MinJ nor DivIVA was therefore required for localization of GFP-MinD at the nascent septal membranes. These findings are consistent with the observation that GFPFtsZ forms discrete structures at fairly regular intervals along cell filaments of minJ-disrupted cells (Bramkamp et al., 2008). Similar distributions have been reported for FtsA, EzrA and ZapA in minJ-disrupted cells, showing that minJ mutations prevent neither the recruitment of these division proteins to FtsZ assemblies nor the formation of septal membranes (Bramkamp et al., 2008).

Two MTSs are involved in the localization of $B$. subtilis MinD at the septal membranes Bacillus subtilis MinD has a sequence at its C-terminus that is predicted to constitute an amphipathic $\alpha$-helix (Szeto et al., 
2002, 2003); the equivalent sequence in $E$. coli may serve in targeting MinD to the membrane and has consequently been designated as the membrane targeting sequence (MTS) (Szeto et al., 2002, 2003; Hu and Lutkenhaus, 2003). Careful inspection of the predicted secondary structure of $B$. subtilis MinD using the Edmundson wheel plot and the PSIPRED and Papia programs revealed that MinD has a second amphipathic $\alpha$-helix close to the previously identified MTS on its N-terminal side (Fig. 2A). We named the second amphipathic $\alpha$-helix (R227-L240) the MTS-like sequence (MTSL) and the previously identified sequence MTS1 (K254-F264). MTSL is predicted to constitute an amphipathic $\alpha$-helix that is rich in basic amino acid residues (four arginine residues) on its polar residue face, which resembles that of MTS1. It would thus seem likely that MTSL is also involved in targeting MinD to membranes. When we looked for the sequence in other organisms, a homologous sequence with a similar amphipathic $\alpha$-helix was found close to MTS1 on its $\mathrm{N}$-terminal side in many other bacterial species (Table 2). In the sequences homologous to MTSL (a 14-residue sequence), at least three basic amino acid residues conserved among Gram-positive bacteria were found (four, if a non-conserved but always basic residue is included),

Table 2. MTSL and conserved regions on the N-terminal side of MTSL of MinD among bacteria, archaea and chloroplasts

\begin{tabular}{|c|c|c|}
\hline \multirow{7}{*}{$\begin{array}{l}\text { Gram-positive } \\
\text { eubacteria }\end{array}$} & Bacillus subtilis & PKNRASIAYRNIARRILGESV \\
\hline & Bacillus cereus & PSGKAALAYRNIARRLLGENV \\
\hline & Listeria innocua & PNNRASQGYRNI ARRILGESI \\
\hline & Clostridium kluyveri & KSAISGQAFRNIARRILGEEV \\
\hline & Clostridium acetobutylicum & QNANAGKAFRDIA \\
\hline & Sporolactobacillus inulinus & PSSKAAMAYRNIGRRILGETV \\
\hline & Sporolactobacillus vineae & PNSKAAQAYRNIGRRILGETV \\
\hline \multirow{6}{*}{$\begin{array}{l}\text { Gram-negative } \\
\text { eubacteria }\end{array}$} & Escherichia coli & INADAGKAYADTVERLLGEER \\
\hline & Salmonella typhimurium & ATADAGKAYADTVDRLLGEER \\
\hline & Yersinia pestis & KESDAGKAYEDTVDRLLGEER \\
\hline & Vibrio cholerae & DQSDAGQAYQDTVARLLGEQV \\
\hline & Pseudomonas aeruginosa & EQSDAGQAYSDAVDRLLGKE I \\
\hline & Neisseria gonorrhoeae & DSVAASEAYKDVIARLLGENR \\
\hline \multirow[t]{2}{*}{ Archaea } & Methanocaldococcus jannaschii & KNSPASQAYMKLASI I AGVPI \\
\hline & Pyrococcus furiosus & PTSPAAIAYKQIAAKLAGIKW \\
\hline \multirow[t]{2}{*}{ Chloroplasts } & Mesostigma viride & KLTLSGISFENAARRLVGRKE \\
\hline & Arabidopsis thaliana & PPTLAGLAFEQAAWRLVEQDS \\
\hline \multirow[t]{2}{*}{ Hyperthermophilic eubacteria } & Aquifex aeolicus & EKFPASQAI IDTARRLMGESI \\
\hline & Thermotoga maritima & GNSRI SKNFENLARRIRGEGV \\
\hline
\end{tabular}

The sequence (P224-V244) that includes the region constituting an $\alpha$-helix designated as MTSL (R227-L240) with flanking sequences at the N-terminal nearest neighbor of the membrane targeting sequence (MTS1) in B. subtilis MinD and the homologous sequences among bacteria, chloroplasts and archaea are shown. Basic, anionic and polar residues are named in black, white and gray boxes, respectively. No specific mark is given to hydrophobic residues, and only residues constituting $\alpha$-helices in the sequence are marked. 
thereby making the net charge of the $\alpha$-helix basic. However, the corresponding sequences among Gram-negative bacteria contain many anionic residues (among which two are conserved) and the net charge of the helix is not basic but acidic. This difference between Gram-positives and Gram-negatives in the net charge of the second amphipathic $\alpha$-helix may reflect distinct functions for the second amphipathic $\alpha$-helix.

To examine the role of the MTSs in the action of MinD, we constructed GFP fusions using the sequence Q252-S268 containing MTS1, the sequence P224-G241 containing MTSL, the sequence of full-length MinD and that of the segment of MinD lacking both MTSs, M1-D223 (this segment is termed $\Delta \mathrm{MTS}$ ). Hereafter, we refer to the localization of the GFP fusions to Q252-S268 and P224-G241 simply as the localization of MTS1 and MTSL, respectively. The genes for the GFP fusions were inserted into the $a m y E$ locus and expressed from the xylose-inducible promoter $\mathrm{P}_{x y l}$ to determine the subcellular locations of the GFP fusions. MinC is reported to be located not only at the septum but also at the poles when overproduced (Gregory et al., 2008). To avoid such artefactual polar localization of GFP-MinD fusions, we induced them at a low xylose concentration of $0.05 \%$. Both MTS1 and MTSL were located at mid-cell sites (Fig. 2D and C, respectively), like full-length $\mathrm{MinD}$ (Fig. 2B), although MTSL was rather diffusely distributed in addition to being enriched in the septal region. Removal of the MTSs from full-length MinD made the septal localization of the MinD segment lacking MTSs ( $\triangle \mathrm{MTS}$ ) less distinct, although it was not completely abolished (Fig. 2E). These results suggest that either MTS is capable of binding to the septal membranes, and that both are thus likely involved in locating MinD to the septal region. These results also suggest that, in addition to the MTSs, some other factor causes MinD to locate to the septal region.

The normal restriction of the location of $\mathrm{MinD}$ to the septum and poles has been reported to be due to direct protein-protein interaction with MinJ (Bramkamp et al., 2008; Patrick and Kearns, 2008). Thus, the possibility that protein-protein interaction with MinJ was responsible for this septal localization of MTSs could not be excluded, even though GFP-MTS (MTS1) binds to membranes in $E$. coli cells (Szeto et al., 2003) that have no MinJ homolog. To investigate this possibility we localized MTS1 and MTSL in minJ mutant B. subtilis cells. Since the localization of MinJ is considered to be controlled by DivIVA, we also localized MTS1 and MTSL in divIVA mutant cells. The phenotype of minJ and divIVA mutants is cell filamentation; in other words, these mutants divide with low frequency and rarely form a septum (Marston et al., 1998; Bramkamp et al., 2008; Patrick and Kearns, 2008). To localize MinD in cells defective in minJ and $\operatorname{divIVA}$, we constructed the double mutants $\min C \min J$ and $\min C \operatorname{divIVA}$, since the filamentation phenotype of minJ and divIVA can be suppressed by disruption of $\operatorname{minC}$ (Marston and Errington, 1999; Patrick and Kearns, 2008), while the minC mutation does not affect the localization of MinD (Marston and Errington, 1999). We found that, even in minJ and divIVA mutant cells, MTSL and MTS1 were located mainly at septa and, infrequently, at the poles, although MTSL was much more diffusely distributed than MTS1 in both minJ and divIVA mutant cells (Fig. 3B and $\mathrm{F}$ for MTSL, and C and G for MTS1, respectively). Removal of both MTSs from full-length MinD made the septal GFP fluorescence disappear (Fig. 3D and $\mathrm{H}$ for minJ and divIVA mutant cells, respectively); we therefore conclude that these MTSs cause MinD to be located at the septum even in the absence of MinJ and DivIVA. The septal localization of the segment of MinD lacking both MTSs ( $\mathrm{MTS}$ ) in wild type host cells, although faint and indistinct (Fig. 2E), and the absence of this septal localization in minJ and divIVA cells (Fig. 3D and $\mathrm{H}$ ) suggest that MinJ interacts with the segment of MinD lacking MTSs to tether the segment to the mid-cell sites.

Since MinD can form oligomers (Suefuji et al., 2002; Lutkenhaus and Sundaramoorthy, 2003; Mileykovskaya et al., 2003; Wu et al., 2011), it is conceivable that the septal localization of GFP-MTSs we observed is due to an interaction of GFP-MTS with endogenous native MinD supplied from a WT allele on the chromosome. To exclude this possibility, we used cells with a $\min D$ dele-

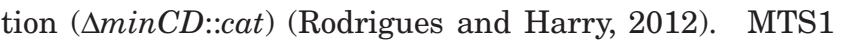
alone was located in the nascent septal regions in the complete absence of native MinD, whereas MTSL was distributed in a quite faint and diffuse manner (Fig. 3. in minJ minCD mutant cells, panels $\mathrm{J}$ and $\mathrm{K}$, for MTSL and MTS1, respectively, and in divIVA minCD mutant cells, panels $\mathrm{M}$ and $\mathrm{N}$ for MTSL and MTS1, respectively). No fluorescent band of GFP-MinD lacking MTSs ( $\triangle \mathrm{MTS}$ ) was detected in the minJ minCD and $\operatorname{divIVA} \min C D$ mutant cells (data not shown). The fluorescence of MTSL was quite faint even at a high level of the inducer $(0.2 \%$ xylose was used for Fig. 3J and M). Thus, it seems reasonable to conclude that MTS1 is targeted to the septal membrane region in cells lacking both endogenous native MinD and either MinJ or DivIVA. The diffuseness and weakness of the fluorescent signal from GFP-MTSL in the cells lacking $\mathrm{MinD}(\min C D)(\mathrm{Fig} .3 \mathrm{~J}$ and $\mathrm{M}$ ), in contrast to the thick fluorescent band of GFP-MTSL in the presence of MinD (Fig. 3B and F), is consistent with an interaction between MTSL and endogenous native MinD, suggesting an involvement of oligomerization (Suefuji et al., 2002; Lutkenhaus and Sundaramoorthy, 2003) with host cell MinD for the septal membrane localization of GFP-labeled MTSL. 


\section{Full length}
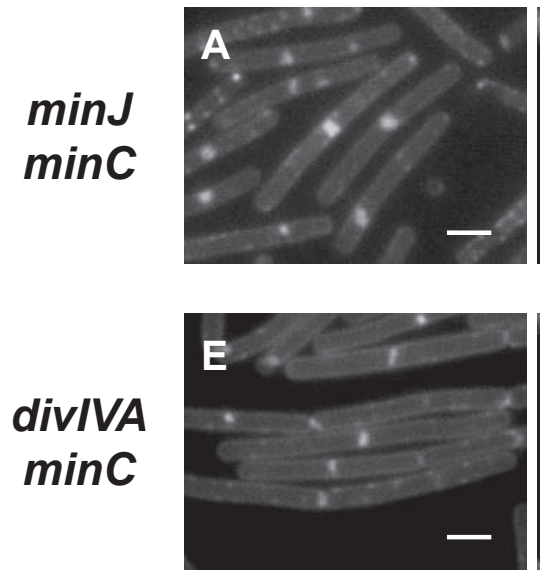

MTSL
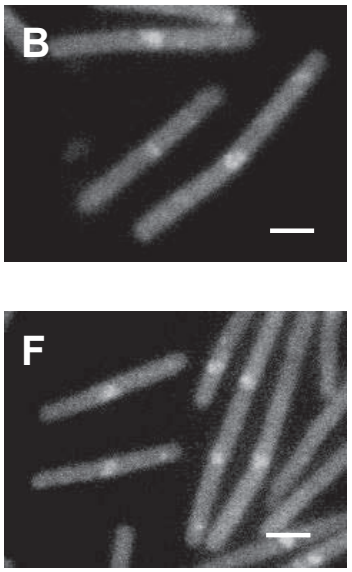

MTS1
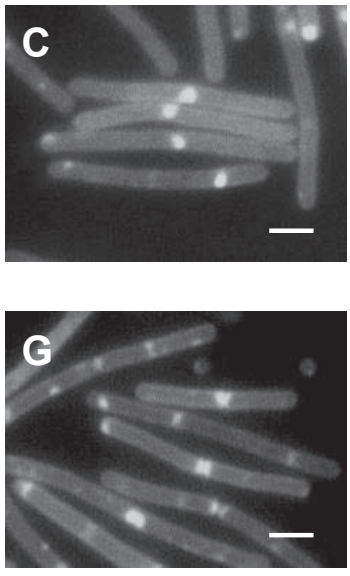

$\Delta \mathrm{MTS}$
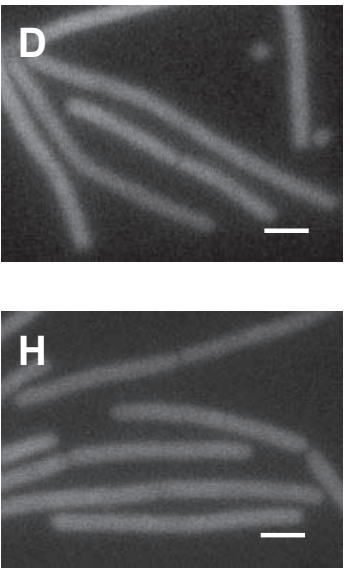

Full length

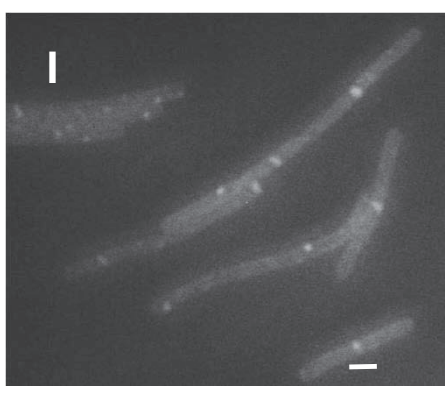

MTSL

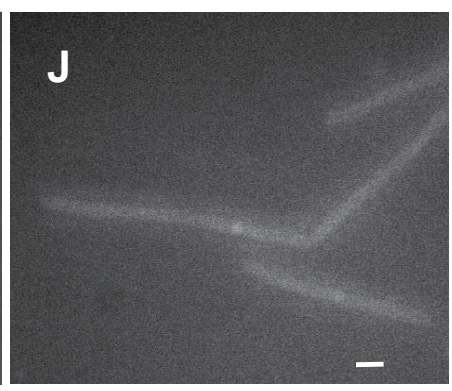

MTS1

\section{minJ \\ $\min C D$}
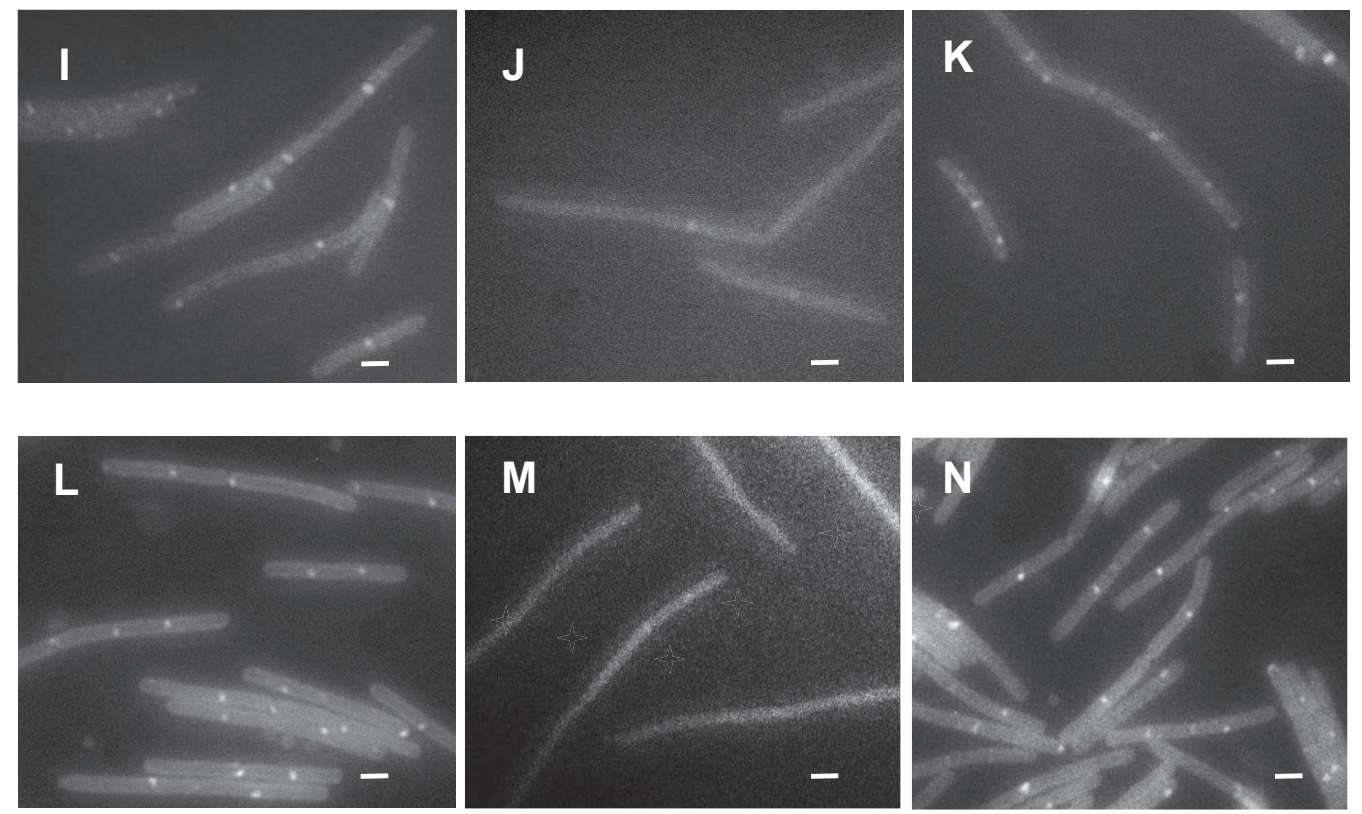

divIVA

$\min C D$

Fig. 3. Localization of MinD (full length), MTSL, MTS1 and a variant of MinD lacking both MTSs ( $\triangle$ MTS) in the mutant cells of $\min J \min C$, divIVA $\min C$, minJ $\min C D$ and divIVA minCD host strains. Bacterial cultivation and fluorescence microscopy of the cellular localization of GFP were conducted under the conditions described for Fig. 2. Host cells used were minJ minC mutant strains (A-D) and divIVA minC mutant strains (E-H). A, GFP-MinD, full length (strain KB267); B, GFP-MTSL (strain KB269); C, GFP-MTS1 (KB270); D, GFP-MinD ${ }^{\triangle M T S}$ (KB268); E, GFP-MinD (KB273); F, GFP-MTSL (KB275); G, GFP-MTS1 (KB276); H, GFP$\operatorname{MinD}^{\Delta \mathrm{MTS}}$ (KB274). Host cells used were minJ minCD mutant strains (I-K) and divIVA minCD mutant strains (L-N). I, GFP-MinD (strain MB511); J, GFP-MTSL (strain MB512); K, GFP-MTS1 (MB513); L, GFP-MinD (MB541); M, GFP-MTSL (MB542); N, GFP-MTS1 (MB543). The level of production of these derivatives was examined by Western blotting with anti-GFP antibody to ascertain that all the constructs produced amounts of GFP fusion proteins almost equal to that of the wild type GFP-MinD, including MinD ${ }^{\Delta \mathrm{MTS}}$. Bars, $2 \mu \mathrm{m}$. In all cultures xylose was included at a concentration of $0.05 \%$, except for $\mathrm{J}$ and $\mathrm{M}$, where $0.2 \%$ xylose was included. Percentages of cells showing septal membrane localization of GFP-labeled variants among the total cells counted (90-230): A, 25\%; B, 29\%; C, $36 \%$; D, 0\%; E, 33\%; F, 29\%; G, 30\%; H, quite faint, $5 \%$. Percentages of cells showing septal localization among minJ minCD mutants (total cells counted, 50-80): I, 41\%; J, quite faint, 9\%; K, 32\%. Percentages of cells showing septal localization among divIVA minCD mutants (total cells counted, 100-170): L, 26\%; M, quite faint, $2 \% ; \mathrm{N}, 24 \%$.

Amphipathic $\alpha$-helix and basic amino acid residues are required for MTS targeting to septal membranes To determine whether the septal localization of the MTSs requires the amphipathic $\alpha$-helical structures, we constructed mutant alleles for GFP-MTSL and GFPMTS1, in which their $\alpha$-helices were disrupted by replace- 
A

\section{MTSL}

RASIAYRNIARRIL

$\downarrow$

RASIAYRNIAPRIL
CCCHEHCCCCCCEE

(Papia)
B

\section{MTS1}

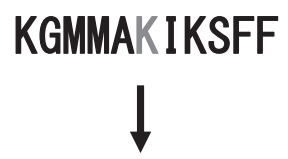

KGMMAPIKSFF CCCHCCECCEE

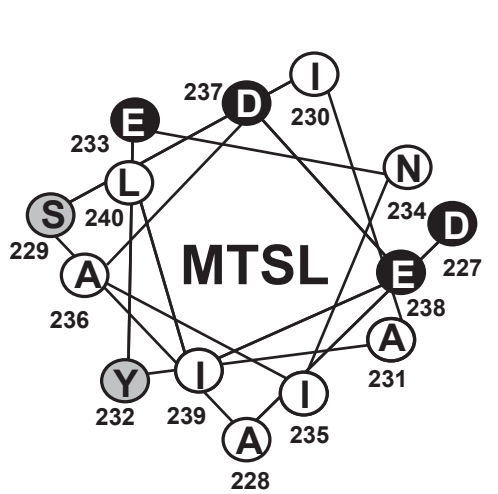

C
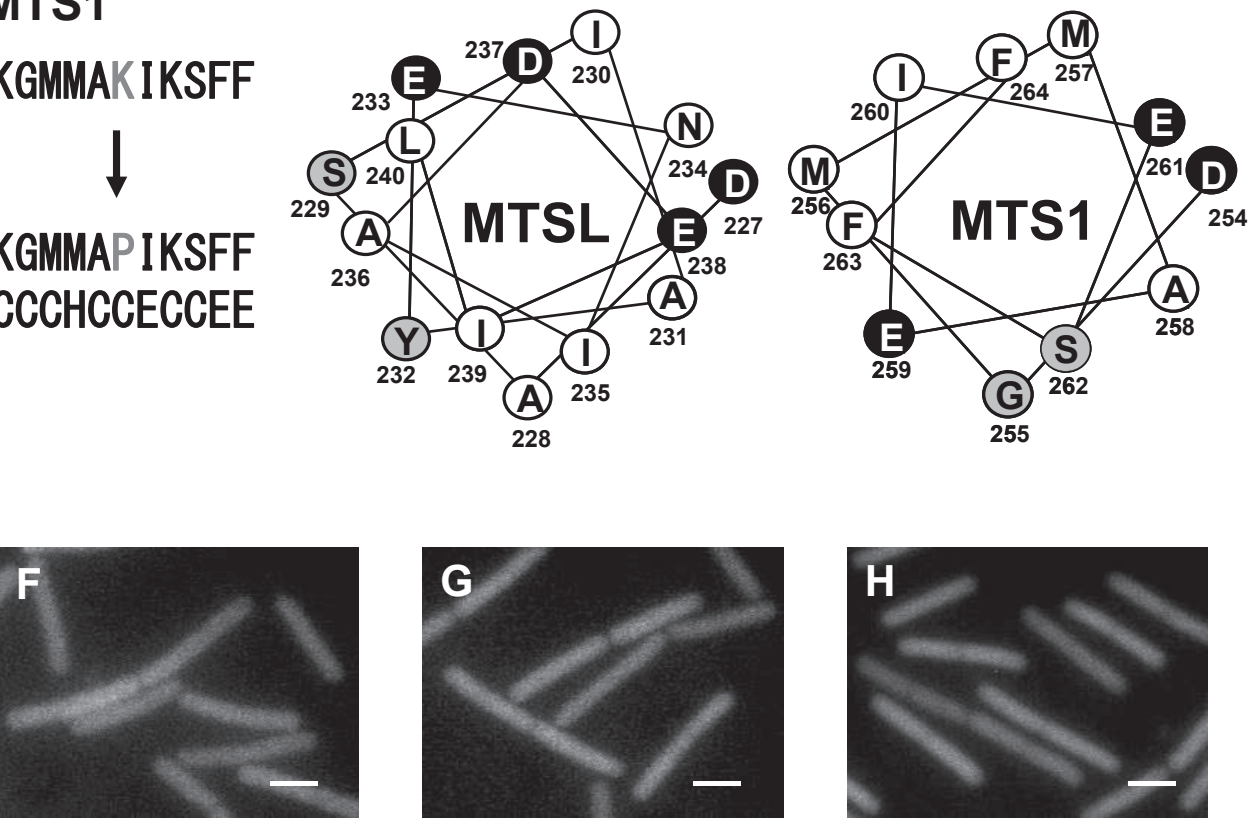

Fig. 4. Localization of mutant MTSs with disrupted helix structures and mutant MTSs in which basic amino acid residues were replaced with acidic residues. Bacterial cultivation and fluorescence microscopy were conducted under the same conditions as for Fig. 2. A, MTSL ${ }^{\text {R237P }}$ and its predicted secondary structure according to the Papia program; B, MTS1 ${ }^{\mathrm{K} 259 \mathrm{P}}$ and its predicted secondary structure; C, MTSL ${ }^{\text {acidic }}$ forms an $\alpha$-helix; D, MTS1 ${ }^{\text {acidic }}$ forms an $\alpha$-helix. In the Edmundson wheel analysis diagrams the characteristics of the amino acid residues are marked as follows: acidic (replaced from basic), polar and hydrophobic residues are in black, gray and white circles, respectively. E, GFP-MTSL ${ }^{\text {R237P }}$ (strain KB261); F, GFP-MTS1 ${ }^{\text {K259P }}$ (strain KB262); G, GFP-MTSL ${ }^{\text {acidic }}$ (KB279); H, GFPMTS1 $^{\text {acidic }}$ (KB283). Bars, $2 \mu \mathrm{m}$. Percentages of cells showing septal membrane localization of GFP-labeled variants among the total cells counted (150-230): E, 0\%; F, 0\%; G, 0\%; H, 0\%.

ment of arginine or lysine residues, at R237 or K259, respectively, with proline; the altered protein is predicted to form a coil structure (Fig. 4A and B). These mutant MTSs, MTSL ${ }^{\text {R237P }}$ and MTS1 ${ }^{\text {K259P }}$, showed a uniformly diffuse distribution in the cytoplasm (Fig. $4 \mathrm{E}$ and F). This result is consistent with previous observations in $E$. coli which show that the correct localization of $E$. coli MinD requires the amphipathic $\alpha$-helical structure within its C-terminus (Szeto et al., 2002; Zhou and Lutkenhaus, 2003). Subsequently, to confirm that the basic residues in the MTSs help to locate them in the membrane, we constructed mutant alleles in which the basic residues of MTSL - R227, R233, R237 and R238 - were replaced with the acidic residues $227 \mathrm{D}, 233 \mathrm{E}, 237 \mathrm{D}$ and $238 \mathrm{E}$ to make MTSL $^{\text {acidic }}$, and the basic residues of MTS1 - K254, K259 and K261 - were replaced with the acidic residues $254 \mathrm{D}$, 259E, and 261D to make MTS1 ${ }^{\text {acidic }}$. These mutant MTSs with acidic residues were still predicted to have $\alpha$-helical structures (Fig. 4C and D). The uniformly diffuse distribution of the fluorescence in the cytoplasm (Fig. 4G and $\mathrm{H}$ ) indicates that the septal localization of the MTSs requires basic residues. We conclude, therefore, that the septal localization of the MTSs requires an amphipathic $\alpha$-helical structure with basic amino acid residues.
To explore further the role of the MTSs in the septal localization of MinD, we localized GFP fusions with various deletion and point mutant derivatives of $\mathrm{MinD}$; these fusions either (1) lacked MTSL $\left(\mathrm{MinD}^{\triangle \mathrm{MTSL}}\right)$ or MTS1 (MinD ${ }^{\triangle \mathrm{MTS} 1}$ ), or (2) contained only the two MTSs (MTSL-MTS1), or (3) had the $\alpha$-helical structure of only MTSL (MinD ${ }^{\text {R237P }}$ ) disrupted, of only MTS1 $\left(\operatorname{MinD}^{\mathrm{K} 259 \mathrm{P}}\right)$, or of both MTSs (MinD ${ }^{\text {R237P/K259P) }}$ (Fig. 5A-F). Five of the derivatives localized to the septal regions, although the fluorescence was somewhat diffuse (Fig. 5A-E), and only the derivative in which both helices were disrupted showed quite weak septal localization and had a low level of fluorescence (MinD ${ }^{\mathrm{R} 237 \mathrm{P} / \mathrm{K} 259 \mathrm{P}}$, Fig. 5F). This weak septal localization of doubly disrupted $\mathrm{MinD}^{\mathrm{R} 237 \mathrm{P} / \mathrm{K} 259 \mathrm{P}}$, which differed from the localization of single MTSL-disruptant MinD $^{\text {R237P }}$ and of single MTS1-disruptant MinD $^{\text {K259P }}$ (Fig. 5D and E, respectively), indicates that an interaction with acidic phospholipids in the membranes by both MTSL and MTS1, as shown by MTSL and MTS1 mutants in Fig. 4, is involved in the septal membrane localization of MinD.

In the case where mutant derivatives were introduced into the minD::pMUTIN strain (Fig. 5J-O), when the derivatives lacked MTSL $\left(\mathrm{MinD}^{\triangle \mathrm{MTSL}}\right)$ or had disrupted 
MTSL (MinD ${ }^{\text {R237P }}$, and both had complete MTS1, there was clear septal localization (Fig. 5J and M). However, $\mathrm{MinD}^{\Delta \mathrm{MTS} 1}$ and $\mathrm{MinD}^{\mathrm{K} 259 \mathrm{P}}$, the derivatives that lacked MTS1 and had disrupted MTS1 (Fig. 5K and N, respectively), both with intact MTSL, lost their capacity to localize to septal membranes in $\min D::$ pMUTIN cells even under high-level induction ( $0.5 \%$ xylose), although they localized septally in $\operatorname{minD}^{+}$cells (Fig. 5B and E). This result indicates that $\mathrm{MinD}$ from the host cell is required for the septal localization of GFP-labeled MinD having MTSL but not MTS1, further suggesting that MTSL contributes to MinD oligomerization.

We also constructed variants of GFP-MinD that had either tandem MTSLs (MinD ${ }^{\triangle \mathrm{MTS} 1}$-MTSL) or tandem MTS1s (MinD ${ }^{\Delta \text { MTSL }}$-MTS1), or in which MTS1 and MTSL replaced each other (MinD ${ }^{\triangle \mathrm{MTSL}}$-MTSL) (Fig. 5G-I). The fusion with tandem MTS1 (Fig. 5H) showed a clear localization in the septal region similar to that of the wild type MinD, but neither fusions of the tandem MTSL nor the MTS1-MTSL exchange showed a clear septal localization (Fig. 5G and I, respectively). When these variants were introduced into the $\min D$ mutant strain induced with $0.05 \%$ xylose, the MinD with tandem MTS1s showed scattered distribution of intense foci with occasional possible septal localization (Fig. 5Q), but MinD with tandem MTSLs did not localize to the septal membrane (Fig. 5P). Thus, tandem duplication of MTS1 caused aberrantly intense foci. The variant with MTS1 and MTSL exchanged showed some weak septal localization (Fig. 5R, $29 \%$ of total cells), and a similarly weak septal signal was found more frequently, in $38 \%$ of total cells, after supplementation of $0.5 \%$ xylose. The correct order of MTSL and MTS1 thus seems to be required for proper septal localization of MinD.

Although the constructed derivatives of GFP-MinD with deletion of either MTS, with tandem MTS1s or tandem MTSLs, or with exchanged MTSs were localized at the septal region, albeit not always clearly, the cells expressing these derivatives exhibited the min phenotype, or the derivatives themselves failed to complement the minicell production phenotype of $\min D$ ::pMUTIN-mutant cells (host strain KB021, Supplementary Fig. S4). Thus, both MTSs are required and they must be in the correct order for MinD to function properly. Examination of the interaction of the mutant derivatives of MinD with MinC using the bacterial adenylate cyclase two-hybrid system
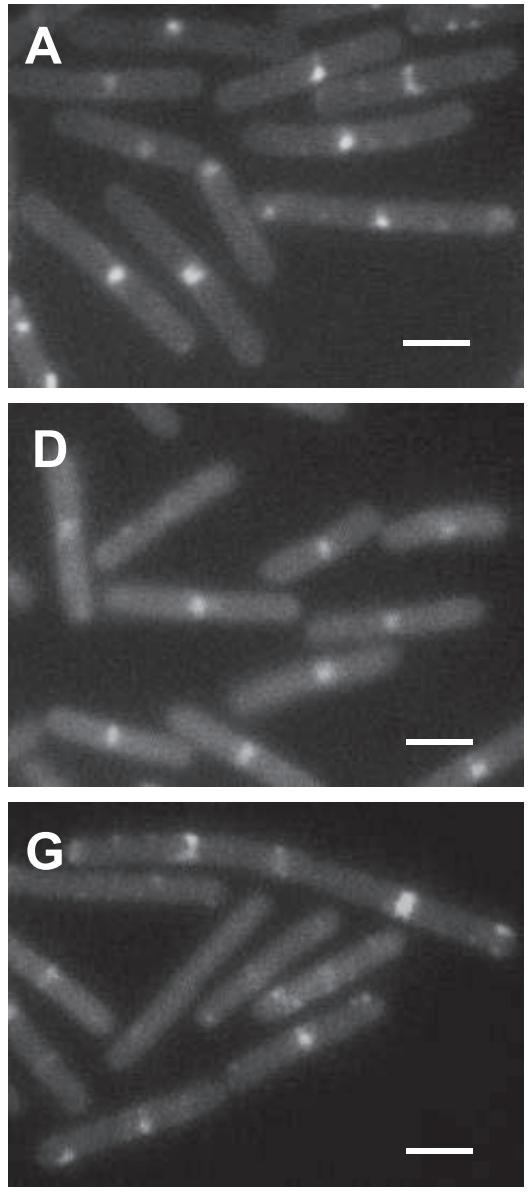
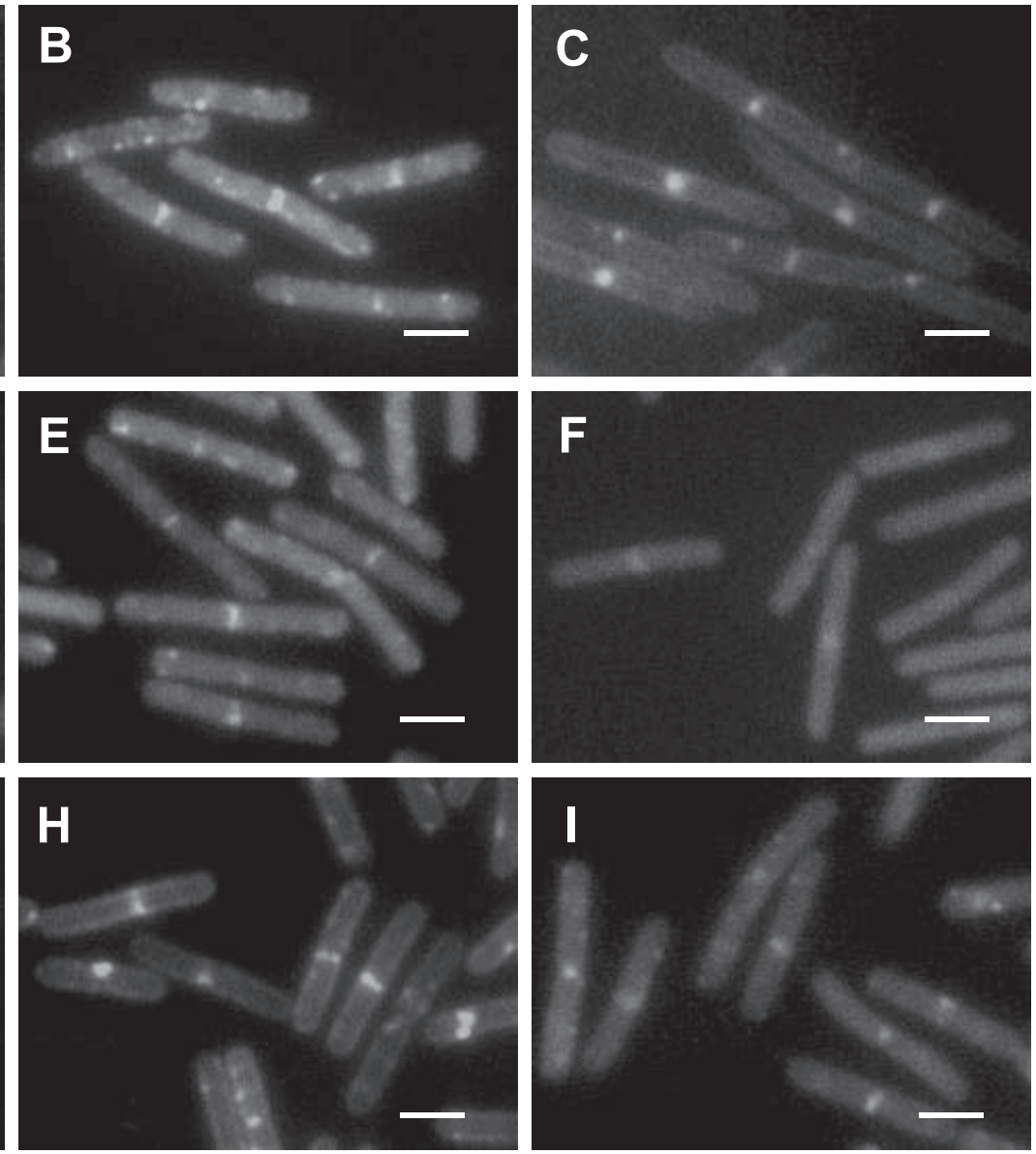

Fig. 5. Continued 

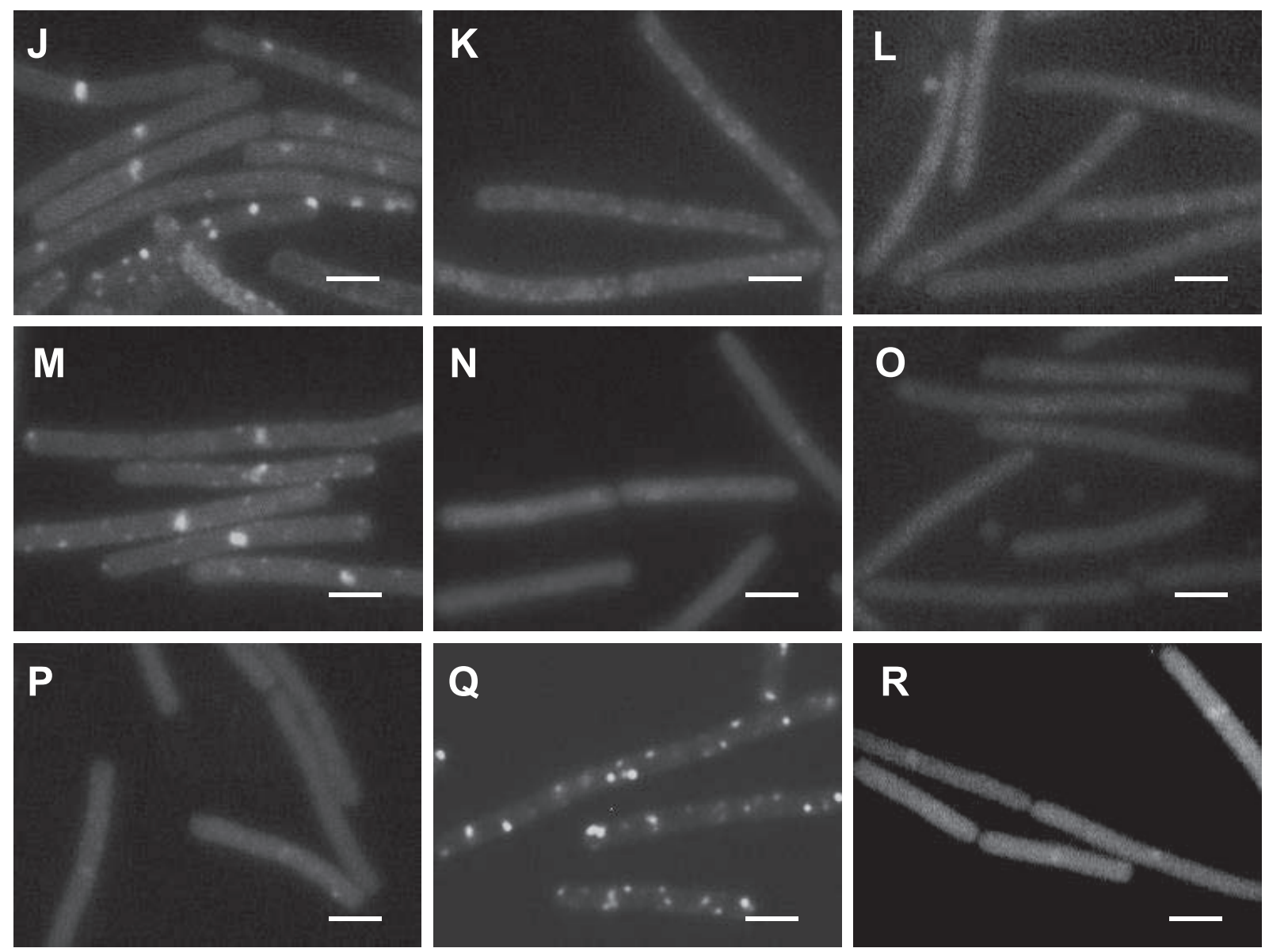

Fig. 5. Localization of MinD with various mutant MTSs in cells of 168 and minD mutant strains. Bacterial cultivation and fluorescence microscopy were conducted under the same conditions as for Fig. 2. In all cultures, xylose was included at a concentration of $0.05 \%$ (panels A-I, and P-R), except for six derivatives of minD mutant strains (panels J-O), where 0.5\% xylose was included. A,

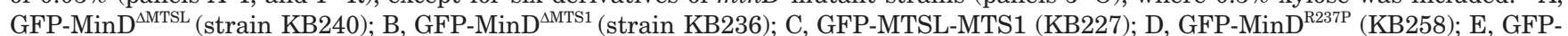
$\operatorname{MinD}^{\text {K259P }}$ (KB259); F, GFP-MinD ${ }^{\text {R237P/K259P }}$ (KB260); G, GFP-MinD ${ }^{\Delta M T S 1}$-MTSL: tandem MTSLs (KB244); H, GFP-MinD ${ }^{\Delta M T S L}$-MTS1: tandem MTS1s (KB246); I, GFP-MinD ${ }^{\triangle M T S L}$-MTSL: exchange of MTS1 and MTSL (KB245). Furthermore, the following minD mutant strains: J, GFP-MinD ${ }^{\Delta \text { MTSL }}$ (KB249); K, GFP-MinD ${ }^{\Delta M T S 1}$ (KB238); L, GFP-MinD ${ }^{\Delta M T S}$ (KB237); M, GFP-MinD ${ }^{\text {R237P }}$ (KB263); N, GFP$\operatorname{MinD}^{\mathrm{K} 259 \mathrm{P}}$ (KB264); O, GFP-MinD ${ }^{\text {R237P/K259P }}$ (KB265); P, GFP-MinD ${ }^{\triangle M T S 1}$-MTSL: tandem MTSL (KB253); Q, GFP-MinD ${ }^{\Delta M T S L}$-MTS1: tandem MTS1s (KB255); R, GFP-MinD ${ }^{\triangle \mathrm{MTSL}}$-MTSL: exchange of MTS1 and MTSL (KB254). Bars, $2 \mu \mathrm{m}$. Percentages of cells showing septal membrane localization of GFP-labeled variants among total cells counted (100-310) in panels A-I were as follows: A, 52\%; B, $38 \%$; K, 25\%; D, 49\%, E, 47\%; F, weak, 8\%; G, 25\%; H, 24\%; I, 28\%. Percentages showing septal membrane localization among the total cells counted (80-260) in panels J-R were as follows: J, 42\%; K, 0\%; L, quite faint, $17 \%$; M, 46\%; N, quite faint, 33\%; O, 0\%; P, faint, $23 \%$; Q, scattered intense foci $98 \%$ and possibly septal $7 \%$; R, weak, $29 \%$. The scattered intense foci observed in the cells of strain KB255 (panel Q) were also observed in cells with another tandem MTS1s strain, KB257, containing a much larger deletion of the MTSL region (data not shown). The products of these $g f p$ fusion genes were examined by Western blotting with anti-GFP antibody and it was confirmed that all the fusion constructs were stable and produced an amount of GFP fusion products almost equal to the amounts produced by GFP fusions to wild type MinD (see Supplementary Fig. S6).

showed that only the wild type full-length MinD interacted with MinC (Supplementary Fig. S5), as expected from the results of the experiments showing the min phenotype.

\section{DISCUSSION}

We found that B. subtilis MinD is located mainly at nascent septal membranes and at an asymmetric site on lateral membranes between the nascent septal mem- branes of filamentous cells of minJ and divIVA mutants (Fig. 1). This result is contrary to the reported MinJand DivIVA-dependent polar and septal localization of MinD (Bramkamp et al., 2008; Patrick and Kearns, 2008). However, MinC and MinD produced by either a low level of induction or expression from its native promoter is located at mid-cell (Gregory et al., 2008); moreover, MinJ produced by expression from its native promoter is also located at mid-cell (van Baarle and Bramkamp, 2010). Here, we have re-confirmed that all 
four components of the Min system, if expressed from their native promoter, are located at the nascent septal membranes of wild type cells (Fig. 1).

Our second finding is that MinD has two amphipathic $\alpha$-helices at its C-terminus, which bind to membranes by partitioning into the membrane bilayer such that the hydrophobic face of the protein is sequestered away from water whereas its polar face can contact the aqueous phase (Johnson and Cornell, 1999; Matsumoto et al., 2015). The weak, and reversible, interaction with membranes of proteins containing amphipathic $\alpha$-helices that serve as MTSs plays an important role in membrane targeting and regulation of protein functions. Representative examples are FtsA, MreB, Noc, DnaA, E. coli MinD and B. subtilis PssA and ClsA (Matsumoto, 1997; Yamaguchi et al., 1999; Pichoff and Lutkenhaus, 2005; Salje et al., 2011; Szwedziak et al., 2012; Adams and Errington, 2015; Matsumoto et al., 2015; Kusaka et al., 2016). In the case of $B$. subtilis MinD a second amphipathic helix, the MTS-like sequence (MTSL), identified in this study, is located close to the previously reported MTS (named MTS1) on its N-terminal side (Szeto et al., 2002, 2003; Hu and Lutkenhaus, 2003). Removal of the two MTSs from MinD (MinD ${ }^{\Delta \mathrm{MTS}}$ ) resulted in a diffuse distribution in the cytoplasm; the MinD localization at nascent septal membranes is thus presumably dependent on the MTSs. Both MTSL and MTS1 were independently localized mainly to the nascent septal membranes - like fulllength MinD (Fig. 2). Septal membrane localization of MTSL was, however, dependent on host cell MinD. MTSL is also therefore considered to be involved in oligomerization (Suefuji et al., 2002; Lutkenhaus and Sundaramoorthy, 2003; Mileykovskaya et al., 2003) of MinD to accomplish the septal localization. When $\mathrm{MinD}^{\Delta \mathrm{MTS}}$ was introduced into mutant cells lacking MinJ or DivIVA, the weak signal that was observed in wild type cells (Fig. 2E) disappeared and diffuse distribution in the cytoplasm was observed (Fig. 3D); this suggests that the MinD domain that interacts with MinJ is probably confined to the segment lacking MTSs.

The synthetic peptide of the MTS of $E$. coli MinD has a highly disordered structure (random coil) and interacts with lipid bilayers containing PG or CL, causing a significant transition to $\alpha$-helix structure, which can be demonstrated by CD spectropolarimetry (Szeto et al., 2003). The transition of, or affinity to, the MTS1 peptide of $B$. subtilis shows essentially the same characteristics with a lipid bilayer containing PG but the transition is less frequent with a bilayer containing CL (Szeto et al., 2003). The mutant GFP-MTSs in which basic amino acid residues were replaced with acidic residues still had a propensity to form $\alpha$-helical structures and caused a uniformly diffuse distribution of GFP fluorescence in the cytoplasm (Fig. 4), indicating that the MTSs require basic amino acid residues in order to localize to septal or mid- cell positions. This result suggests that the interaction of MTSs with acidic phospholipids in the membrane is essential for the correct localization of MinD. In other words, the acidic phospholipids in the membranes probably contribute greatly to the septal membrane localization of MinD in B. subtilis cells.

Studies with liposomes have demonstrated that the binding of MinD to the membrane depends on membrane lipid composition, with a high affinity to acidic over zwitterionic phospholipids (Mileykovskaya et al., 2003). MinD moves abnormally in focal clusters and cannot oscillate normally in mutant $E$. coli cells that lack PE and contain only acidic phospholipids; this suggests that the membrane binding of MinD depends on lipid composition, which can change the protein retention time on the membrane (Mileykovskaya et al., 2003; Mileykovskaya and Dowhan, 2005). The fact that GFPMinD oscillations similar to those in wild type $E$. coli cells occur in mutant cells that lack both PG and CL is probably due to the accumulated phosphatidic acid, which is the precursor of the major phospholipids (Mileykovskaya et al., 2009). In B. subtilis cells, such domains rich in acidic phospholipids are located at mid-cell and polar membranes (Kawai et al., 2004, 2006; Matsumoto et al., 2006; Mileykovskaya and Dowhan, 2009; Matsumoto et al., 2015). The direct substrate of CL synthesis, PG, is assumed to be located in the septal membranes as well (Kusaka et al., 2016), since the enzyme phosphatidylglycerophosphate synthase, which works at the committed step for PG synthesis, is located at the septal membrane (Nishibori et al., 2005; Matsumoto et al., 2006) in addition to the septally located CL synthase (Norris et al., 2015; Kusaka et al., 2016). The nascent septal membrane itself, which is rich in acidic phospholipids and probably also in PG (Matsumoto et al., 2015), is thus expected to have an essential role in recruiting $\mathrm{MinD}$ and facilitating its correct action, since $B$. subtilis MinD MTS1 has a higher affinity to PG (Szeto et al., 2003).

Why does B. subtilis MinD have two MTSs containing amphipathic $\alpha$-helices with basic amino acid residues? The answer to this question may lie in the different behavior of MinD in $E$. coli and B. subtilis. Whereas $E$. coli MinD rapidly oscillates from one cell pole to the opposite one, B. subtilis MinD is stably located at the mid-cell sites or active cell division sites. Bacillus subtilis MinC is also located primarily at the mid-cell sites and, more transiently, at the new poles (recently completed septa), and, probably in association with MinD, moves from the new poles to mid-cell and apparently rotates around the septum (Gregory et al., 2008). Although the Min system was thought to act specifically on FtsZ assembly, the system is inactive at the mid-cell division site; moreover, it may block the division process downstream of FtsZ at the new poles. At the poles, MinCD destabilizes the FtsZ assembly, thereby allowing FtsZ to relocate 
to mid-cell more efficiently and preventing an aberrant secondary FtsZ-ring formation adjacent to the new cell poles (Bramkamp et al., 2008; Gregory et al., 2008; van Baarle and Bramkamp, 2010). To explain the complex movements and activity of MinCD in the B. subtilis Min system, we propose that the pair of amphipathic $\alpha$-helices with basic residues at the C-terminus of MinD plays a major role, since they would have a higher affinity to acidic phospholipids present at mid-cell and in the poles. The distribution and action of the two MTSs is different; MTS1 associates tightly with the septal membranes, while MTSL is diffused within a region close to the mid-cell membranes and its association to the membranes depends on another MinD molecule. MTSL may thus play a role in cooperative binding of MinD to the septal membranes, which are rich in acidic phospholipids.

Although the localization of MinD is believed to be regulated by MinJ and DivIVA, our results show that MinD is located at mid-cell sites even in mutant cells lacking MinJ and DivIVA. The formation of discrete structures of MinD-associated nascent septal membranes at fairly regular intervals in the filamentous cells of the minJ-disrupted mutant (Fig. 1 and Supplementary Fig. $\mathrm{S} 1$ ) is consistent with the formation by FtsZ of discrete structures at fairly regular intervals along filaments of minJ-disrupted cells (Bramkamp et al., 2008). Similar patterns have been observed for the early division proteins FtsA, EzrA and ZapA in minJ-disrupted filamentous cells, showing that minJ disruption does not prevent the recruitment of these division proteins to FtsZ assemblies (nor the formation of foci of lipid membrane that are stained by Nile Red) (Bramkamp et al., 2008). Targeting of MinJ to the mid-cell division sites depends on a late division protein ( $\mathrm{PBP}-2 \mathrm{~B}$, a penicillin-binding protein essential for the synthesis of cell wall peptidoglycan) (Bramkamp et al., 2008). Therefore, it seems that the mid-cell localization of MinJ depends on the assembly of most of the division machinery (Bramkamp et al., 2008). This is consistent with the completely interdependent and concerted assembly in two steps of the division apparatus for the division proteins (Errington et al., 2003; Gamba et al., 2009), in which septally located lipid synthetic enzymes are involved (Nishibori et al., 2005; Matsumoto et al., 2006, 2012, 2015; Takada et al., 2014). In addition, MinC is a late recruit to the mid-cell division site as it arrives after the early division protein EzrA and DivIVA, which is responsible for locating MinC at the division sites (Gregory et al., 2008), contrary to the widely held view of the action of the Min system. Thus, both early and late division proteins are likely recruited to the division sites to assemble most of the division machinery, followed by DivIVA, then MinJ, and ultimately MinCD (Bramkamp et al., 2008). Another prediction of this new model is that the first step of division site selection is effected not by the proteins noted above but probably by the alternative system of nucleoid occlusion, which prevents FtsZ-rings from constricting over unsegregated nucleoids (Errington et al., 2003; Wu and Errington, 2004; Rodriguez and Harry, 2012; Rowlett and Margolin, 2015). The fact that foci of GFP-MinD are located in the inter-nucleoid space (Supplementary Fig. S2) suggests that nucleoid occlusion occurs as the first step of division site selection in minJ and divIVA mutant cells.

In the model we propose, the mid-cell-assembled divisome machinery, which includes early and late divisome proteins and the enzymes for the synthesis of murein and lipids, would form an arc of peptidoglycan and membrane lipids that can be stained with FM 4-64. The acidic phospholipids in the membrane that are newly synthesized in the arc of the divisome machinery would then contribute to recruiting MinD through its MTSs to the membrane; the protein-protein interaction of the segment in the middle of MinD with MinJ would constitute a separate mechanism. MinD might be recruited soon after the initiation of the synthesis of membrane lipids, since acidic phospholipid (PG) synthesis might exceed PE synthesis, as observed in E. coli membranes (Shibuya, 1992; Matsumoto, 1997). In spite of the recruitment of MinD to the mid-cell division site in the minJ mutant cells, the mutant cells are filamentous, indicating that the divisome machinery is severely perturbed and unable to bind or recruit MinC. This is probably because MinD is not fully assembled in the machinery and/or because the divisome is in an abnormal state due to the lack of indispensable division components in the minJ mutant cells; these components may include FtsA, EzrA, FtsL and PBP2B with which MinJ interacts directly (Bramkamp et al., 2008).

We thank Professors Emeriti Isao Shibuya and Yoshito Sadaie and Eugenia Mileykovskaya for their continuous encouragement, and Victor Norris for critical reading of this manuscript. Thanks are also due to Junji Uchiyama, Michihiro Hashimoto, Takatsugu Sato and Yukiko Imai for discussion. We also thank Elizabeth J. Harry, Daniel B. Kearns, Kei Asai and Peter Lewis for generous gifts of bacterial strains and plasmids. This work was supported in part by Grants-in-Aid for Scientific Research from the Ministry of Education, Culture, Sports, Science and Technology of Japan.

\section{REFERENCES}

Aarsman, M. E. G., Piette, A., Fraipont, C., Vinkenvleugel, T. M. F., Nguyen-Distèche, M., and den Blaauwen, T. (2005) Maturation of the Escherichia coli divisome occurs in two steps. Mol. Microbiol. 55, 1631-1645.

Adams, D. W., Wu, L. J., and Errington, J. (2015) Nucleoid occlusion protein Noc recruit to the bacterial membrane. EMBO J. 34, 491-501.

Anagnostopoulos, C., and Crawford, I. P. (1961) Transformation studies on the linkage of markers in the tryptophan pathway in Bacillus subtilis. Proc. Natl. Acad. Sci. USA 47, 378-390. 
Bernhardt, T. G., and de Boer, P. A. J. (2005) SlmA, a nucleoidassociated, FtsZ binding protein required for blocking septal ring assembly over chromosomes in E. coli. Mol. Cell 18, 555-564.

Bi, E., and Lutkenhaus, J. (1991) FtsZ ring structure associated with division in Escherichia coli. Nature 354, 161-164.

Bramkamp, M., Emmins, R., Weston, L., Donovan, C., Daniel, R. A., and Errington, J. (2008) A novel component of the division-site selection system of Bacillus subtilis and a new mode of action for the division inhibitor MinCD. Mol. Microbiol. 70, 1556-1569.

Dajkovic, A., Lan, G., Sun, S. X., Wirtz, D., and Lutkenhaus, J. (2008) MinC spatially controls bacterial cytokinesis by antagonizing the scaffolding function of FtsZ. Curr. Biol. 18, 235-244.

de Boer, P. A. J., Crossley, R. E., and Rothfield, L. I. (1989) A division inhibitor and a topological specificity factor coded for by the minicell locus determine proper placement of the division septum in E. coli. Cell 56,641-649.

de Boer, P. A. J., Crossley, R. E., and Rothfield, L. I. (1990) Central role for the Escherichia coli minC gene product in two different cell division- inhibition systems. Proc. Natl. Acad. Sci. USA 87, 1129-1133.

Errington, J., Daniel, R. A., and Scheffers, D. J. (2003) Cytokinesis in bacteria. Microbiol. Molec. Biol. Rev. 67, 52-65.

Fu, X., Shih, Y. L., Zhang, Y., and Rothfield, L. I. (2001) The MinE ring required for proper placement of the division site is a mobile structure that changes its cellular location during the Escherichia coli division cycle. Proc. Natl. Acad. Sci. USA 98, 980-985.

Gamba, P., Veening, J. W., Saunders, N. J., Hamoen, L. W., and Daniel, R. A. (2009) Two-step assembly dynamics of the Bacillus subtilis divisome. J. Bacteriol. 191, 4186-4194.

Gregory, J. A., Becker, E. C., and Pogliano, K. (2008) Bacillus subtilis MinC destabilizes FtsZ-rings at new cell poles and contributes to the timing of cell division. Genes Dev. 22, $3475-3488$.

$\mathrm{Hu}, \mathrm{Z}$., and Lutkenhaus, J. (2001) Topological regulation of cell division in $E$. coli: spatiotemporal oscillation of MinD requires stimulation of its ATPase by MinE and phospholipid. Mol. Cell 7, 1337-1343.

$\mathrm{Hu}, \mathrm{Z}$., and Lutkenhaus, J. (2003) A conserved sequence at the C-terminus of MinD is required for binding to the membrane and targeting MinC to the septum. Mol. Microbiol. 47, 345-355.

Johnson, J. E., and Cornell, R. B. (1999) Amphitropic proteins: regulation by reversible membrane interactions. Mol. Membr. Biol. 16, 217-235.

Karimova, G., Dautin, N., and Ladant, D. (2005) Interaction network among Escherichia coli membrane proteins involved in cell division as revealed by bacterial two-hybrid analysis. J. Bacteriol. 187, 2233-2243.

Kawai, F., Shoda, M., Harashima, R., Sadaie, Y., Hara, H., and Matsumoto, K. (2004) Cardiolipin domains in Bacillus subtilis Marburg membranes. J. Bacteriol. 186, 1475-1483.

Kawai, F., Hara, H., Takamatsu, H., Watabe, K., and Matsumoto, K. (2006) Cardiolipin enrichment in spore membranes and its involvement in germination of Bacillus subtilis Marburg. Genes Genet. Syst. 81, 69-76.

Kobayashi, K., Ehrlich, D. S., Albertini, A., Amati, G., Andersen, K. K., Arnaud, M., Asai, K., Ashikaga, S., Aymerich, S., Bessieres, P., et al. (2003) Essential Bacillus subtilis genes. Proc. Natl. Acad. Sci. USA 100, 4678-4683.

Kusaka, J., Shuto, S., Imai, Y., Ishikawa, K., Saito, T., Natori, K., Matsuoka, S., Hara, H., and Matsumoto, K. (2016)
Septal localization by membrane targeting sequences and a conserved sequence essential for activity at the $\mathrm{COOH}-$ terminus of Bacillus subtilis cardiolipin synthase. Res. Microbiol. 167, 202-214.

Lenarcic, R., Halbedel, S., Visser, L., Shaw, M., Wu, L. J., Errington, J., Marenduzzo, D., and Hamoen, L. W. (2009) Localisation of DivIVA by targeting to negatively curved membranes. EMBO J. 28, 2272-2282.

Lewis, P. J., and Marston, A. L. (1999) GFP vectors for controlled expression and dual labeling of protein fusions in Bacillus subtilis. Gene 227, 101-109.

Lutkenhaus, J., and Sundaramoorthy, M. (2003) MinD and role of the deviant Walker A motif, dimerization and membrane binding in oscillation. Mol. Microbiol. 48, 295-303.

Lutkenhaus, J. (2007) Assembly dynamics of the bacterial MinCDE system and spatial regulation of the $\mathrm{Z}$ ring. Annu. Rev. Biochem. 76, 539-562.

Marston, A. L., and Errington, J. (1999) Selection of the midcell division site in Bacillus subtilis through MinD-dependent polar localization and activation of MinC. Mol. Microbiol. 33, 84-96.

Marston, A. L., Thomaides, H. B., Edwards, D. H., Sharpe, M. E., and Errington, J. (1998) Polar localization of the MinD protein of Bacillus subtilis and its role in selection of the mid-cell division site. Genes Dev. 12, 3419-3430.

Matsumoto, K. (1997) Phosphatidylserine synthase from bacteria. Biochim Biophys Acta 1348, 214-217.

Matsumoto, K., Matsuoka, S., and Hara, H. (2012) Membranes and lipids. In Escherichia coli and Bacillus subtilis: The Frontiers of Molecular Microbiology Revisited. (eds.: Sadaie, Y., and Matsumoto, K.), pp. 61-91. Research Signpost, Kerala, India.

Matsumoto, K., Hara, H., Fishov, I., Mileykovskaya, E., and Norris, V. (2015) The membrane: transertion as an organizing principle in membrane heterogeneity. Front. Microbiol. 6, 572 .

Matsumoto, K., Kusaka, J., Nishibori, A., and Hara, H. (2006) Lipid domains in bacterial membranes. Mol. Microbiol. 61, 1110-1117.

Meinhardt, H., and de Boer, P. A. J. (2001) Pattern formation in Escherichia coli: a model for the pole-to-pole oscillations of Min proteins and the localization of the division site. Proc. Natl. Acad. Sci. USA 98, 14202-14207.

Mileykovskaya, E., and Dowhan, W. (2000) Visualization of phospholipid domains in Escherichia coli by using the cardiolipin-specific fluorescent dye $10-N$-nonyl acridine orange. J. Bacteriol. 182, 1172-1175.

Mileykovskaya, E., Fishov, I., Fu, X., Corbin, B. D., Margolin, W., and Dowhan, W. (2003) Effects of phospholipid composition on MinD-membrane interactions in vitro and in vivo. J. Biol. Chem. 278, 22193-22198.

Mileykovskaya, E., and Dowhan, W. (2005) Role of membrane lipids in bacterial division-site selection. Curr. Opin. Microbiol. 8, 136-142.

Mileykovskaya, E., and Dowhan, W. (2009) Cardiolipin membrane domains in prokaryotes and eukaryotes. Biochim. Biophys. Acta 1788, 2084-2091.

Mileykovskaya, E., Ryan, A. C., Mo, X., Lin, C. C., Khalaf, K. I., Dowhan, W., and Garrett, T. A. (2009) Phosphatidic acid and $N$-acylphosphatidyl ethanolamine form membrane domains in Escherichia coli mutant lacking cardiolipin and phosphatidylglycerol. J. Biol. Chem. 284, 2990-3000.

Nishibori, A., Kusaka, J., Hara, H., Umeda, M., and Matsumoto, K. (2005) Phosphatidylethanolamine domains and localization of phospholipid synthases in Bacillus subtilis mem- 
branes. J. Bacteriol. 187, 2163-2174.

Norris, V., Mileykovskaya, E., and Matsumoto, K. (2015) Extending the transertion hypothesis. Biochem. Anal. Biochem. 4, 1000234 .

Patrick, J. E., and Kearns, D. B. (2008) MinJ (YvjD) is a topological determinant of cell division in Bacillus subtilis. Mol. Microbiol. 70, 1166-1179.

Pichoff, S., and Lutkenhaus, J. (2005) Tethering the Z ring to the membrane through a conserved membrane targeting sequence in FtsA. Mol. Microbiol. 55, 1722-1734.

Ramamurthi, K. S., and Losick, R. (2009) Negative membrane curvature as a cue for subcellular localization of a bacterial protein. Proc. Natl. Acad. Sci. USA 106, 13541-13545.

Raskin, D. M., and de Boer, P. A. J. (1999) MinDE-Dependent poleto-pole oscillation of division inhibitor MinC in Escherichia coli. J. Bacteriol. 181, 6419-6424.

Rodrigues, C. D. A., and Harry, E. J. (2012) The Min system and nucleoid occlusion are not required for identifying the division site in Bacillus subtilis but ensure its efficient utilization. PLoS Genet. 8, e1002561.

Rowlett, V. W., and Margolin, W. (2015) The Min system and other nucleoid-independent regulators of $\mathrm{Z}$ ring positioning. Front Microbiol. 6, 478.

Salje, J., van den Ent, F., de Boer, P., and Löwe, J. (2011) Direct membrane binding by bacterial actin MreB. Mol. Cell 43, 478-487.

Sambrook, J., Fritsch, E. F., and Maniatis, T. (1989) Molecular Cloning: ALaboratory Manual (2nd ed.), Cold Spring Harbor Laboratory Press, New York.

Scheffers, D. J. (2008) The effect of MinC on FtsZ polymerization is $\mathrm{pH}$ dependent and can be counteracted by ZapA. FEBS Lett. 582, 2601-2608.

Shibuya, I. (1992) Metabolic regulations and biological functions of phospholipids in Escherichia coli. Prog. Lipid Res. 31, $245-299$.

Suefuji, K., Valluzzi, R., and RayChaudhuri, D. (2002) Dynamic assembly of MinD into filament bundles modulated by ATP, phospholipids, and MinE. Proc. Natl. Acad. Sci. USA 99, 16776-16781.

Szeto, T. H., Rowland, S. L., Rothfield, L. I., and King, G. F. (2002)
Membrane localization of MinD is mediated by a C-terminal motif that is conserved across eubacteria, archaea, and chloroplasts. Proc. Natl. Acad. Sci. USA 99, 15693-15698.

Szeto, T. H., Rowland, S. L., Habrukowich, C. L., and King, G. F. (2003) The MinD membrane targeting sequence is a transplantable lipid-binding helix. J. Biol. Chem. 278, 4005040056.

Szwedziak, P., Wang, Q., Freund, S. M. V., and Löwe, J. (2012) FtsA forms actin-like protofilaments. EMBO. J. 31, 22492260.

Takada, H., Fukushima-Tanaka, S., Morita, M., Kasahara, Y., Watanabe, S., Chibazakura, T., Hara, H., Matsumoto, K., and Yoshikawa, H. (2014) An essential enzyme for phospholipid synthesis associates with the Bacillus subtilis divisome. Mol. Microbiol. 91, 242-255.

Takamatsu, H., Kodama, T., Imamura, A., Asai, K., Kobayashi, K., Nakayama, T., Ogasawara, N., and Watabe, K. (2000) The Bacillus subtilis yabG gene is transcribed by sigK RNA polymerase during sporulation, and yabG. J. Bacteriol. 182, 1883-1888.

van Baarle, S., and Bramkamp, M. (2010) The MinCDJ system in Bacillus subtilis prevents minicell formation by promoting divisome disassembly. PLoS One 5, e9850.

Wu, L. J., and Errington, J. (2004) Coordination of cell division and chromosome segregation by a nucleoid occlusion protein in Bacillus subtilis. Cell 117, 915-925.

Wu, W., Park, K.-T., Holyoak, T., and Lutkenhaus, J. (2011) Determination of structure of the MinD-ATP complex reveals the orientation of MinD on the membrane and the relative location of the binding sites for MinE and MinC. Mol. Microbiol. 79, 1515-1528

Yamaguchi, Y., Hase, M., Makise, M., Miwa, S., Yoshimi, T., Ishikawa, Y., Tsuchiya, T., and Mizushima, T. (1999) Involvement of Arg-328, Arg-334 and Arg-342 of DnaA protein in the functional interaction with acidic phospholipids. Biochem. J. 340, 433-438.

Zhou, H., and Lutkenhaus, J. (2003) Membrane binding by MinD involves insertion of hydrophobic residues within the C-terminal amphipathic helix into the bilayer. J. Bacteriol. 185, 4326-4335. 